\title{
Hypoxia-induced microRNA-424 expression in human endothelial cells regulates HIF- $\alpha$ isoforms and promotes angiogenesis
}

\author{
Goutam Ghosh,, ${ }^{1}$ dira V. Subramanian,, ${ }^{2}$ Neeta Adhikari, ${ }^{3}$ Xiaoxiao Zhang, ${ }^{1}$ Hemant P. Joshi, ${ }^{1}$ \\ David Basi, ${ }^{4}$ Y.S. Chandrashekhar, ${ }^{5}$ Jennifer L. Hall, ${ }^{3}$ Sabita Roy, ${ }^{1,6}$ \\ Yan Zeng, ${ }^{1}$ and Sundaram Ramakrishnan ${ }^{1,2,7}$

\begin{abstract}
1Department of Pharmacology, ${ }^{2}$ Department of Obstetrics and Gynecology, ${ }^{3}$ Lillehei Heart Institute, Division of Cardiology, Department of Medicine, ${ }^{4}$ Department of Developmental and Surgical Science, School of Dentistry, ${ }^{5}$ Department of Medicine, VA Medical Center, ${ }^{6}$ Department of Surgery, and
\end{abstract} \\ ${ }^{7}$ Masonic Comprehensive Cancer Center, University of Minnesota, Minneapolis, Minnesota, USA.
}

\begin{abstract}
Adaptive changes to oxygen availability are critical for cell survival and tissue homeostasis. Prolonged oxygen deprivation due to reduced blood flow to cardiac or peripheral tissues can lead to myocardial infarction and peripheral vascular disease, respectively. Mammalian cells respond to hypoxia by modulating oxygen-sensing transducers that stabilize the transcription factor hypoxia-inducible factor $1 \alpha(\mathrm{HIF}-1 \alpha)$, which transactivates genes governing angiogenesis and metabolic pathways. Oxygen-dependent changes in HIF-1 $\alpha$ levels are regulated by proline hydroxylation and proteasomal degradation. Here we provide evidence for what we believe is a novel mechanism regulating HIF-1 $\alpha$ levels in isolated human ECs during hypoxia. Hypoxia differentially increased microRNA-424 (miR-424) levels in ECs. miR-424 targeted cullin 2 (CUL2), a scaffolding protein critical to the assembly of the ubiquitin ligase system, thereby stabilizing HIF- $\alpha$ isoforms. Hypoxia-induced miR-424 was regulated by PU.1-dependent transactivation. PU.1 levels were increased in hypoxic endothelium by RUNX-1 and C/EBP $\alpha$. Furthermore, miR-424 promoted angiogenesis in vitro and in mice, which was blocked by a specific morpholino. The rodent homolog of human miR-424, mu-miR-322, was significantly upregulated in parallel with HIF-1 $\alpha$ in experimental models of ischemia. These results suggest that miR-322/424 plays an important physiological role in post-ischemic vascular remodeling and angiogenesis.
\end{abstract}

\section{Introduction}

Reduced oxygen availability affects cells and tissues during normal embryonic development, wound healing, and physical exertion as well as during pathological conditions such as myocardial infarction, stroke, and cancer. As a consequence, a complex signaling system is activated in cells to overcome hypoxia to restore oxygen and nutrient homeostasis. Angiogenesis is critical to this response, and vascular ECs constitute the first-line interface with blood to detect changes in oxygen levels (1). One of the immediate responses to hypoxia is transcriptional activation of several genes leading to angiogenesis and metabolic adaptation. A central mediator of this transcriptional activation is the hypoxia-inducible factor (HIF) family of proteins. HIF- 1 is composed of 2 subunits, HIF- $1 \alpha$ and HIF-1 $\beta$. HIF1- $\alpha$ levels are maintained at lower levels under normoxia via proteasomal degradation. A reduction in oxygen levels inhibits the degradation of HIF1- $\alpha$, which then heterodimerizes with HIF1- $\beta$ (which is constitutively expressed) and translocates to the nucleus (2). HIF1- $\alpha / \beta$ dimer binds to hypoxia response elements and transactivates target genes involved in cellular metabolism, angiogenesis, and erythropoiesis (3).

Modulation of HIF1- $\alpha$ levels therefore constitutes the ratelimiting step in hypoxic response. Under normoxic conditions, HIF1- $\alpha$ is hydroxylated at 2 proline residues, $\mathrm{P}^{402}$ and $\mathrm{P}^{564}$, by prolyl hydroxylase domain (PHD) proteins (4). Proline hydroxyl-

Authorship note: Goutam Ghosh and Indira V. Subramanian contributed equally to this work.

Conflict of interest: The authors have declared that no conflict of interest exists. Citation for this article: J Clin Invest. 2010;120(11):4141-4154. doi:10.1172/JCI42980. ated HIF1- $\alpha$ is recognized by von Hippel-Lindau (VHL) protein with the VHL $\beta$ domain, while its $\alpha$ domain binds elongin $\mathrm{C}(5,6)$. Elongin $\mathrm{C}$, in turn, binds elongin $\mathrm{B}$, a ubiquitin-like protein, and together elongin B/C bridge VHL to cullin 2 (CUL2), a scaffolding protein. VHL, elongin B/C, and CUL2 associate via several proteinprotein interactions with RING-box protein (RBX1) to form the VCBCR complex, an E3 ubiquitin ligase complex (7). HIF1- $\alpha$ is then rapidly degraded by $26 \mathrm{~S}$ proteasome following polyubiquitination on lysine residues by the VCBCR complex. In addition to protein stability, HIF1- $\alpha$ is also transcriptionally regulated (8). In the present study, we provide evidence for a tertiary level of control by microRNA (miRNA) regulating the proteasomal degradation pathway of HIF1- $\alpha$. miRNAs are small regulatory RNA molecules of 21-23 nucleotides in length that posttranscriptionally modulate translation of target mRNA by interacting with the $3^{\prime}$ untranslated region (9-11). Differential expression of miRNA under hypoxia was determined in a number of cell types including ECs, smooth muscle cells, and tumor cell lines. We found that miR-210 is ubiquitously upregulated in all the different cell types under hypoxic conditions. However, miR-424 was differentially upregulated in the cells associated with the vasculature. Therefore, we investigated the role of miR-424 in ECs and angiogenesis. Our studies show that miR-424 was upregulated in hypoxic ECs and in ischemic tissues undergoing vascular remodeling. miR-424 targeted CUL2 to destabilize the E3-ligase assembly, thereby increasing HIF-1 $\alpha$ levels. Furthermore, we found that miR-424 expression was regulated by C/EBP- $\alpha /$ RUNX-1-mediated transactivation of PU.1. These studies identify what we believe is a new pathway regulating the levels of HIF-1 $\alpha$ and the angiogenic response. 

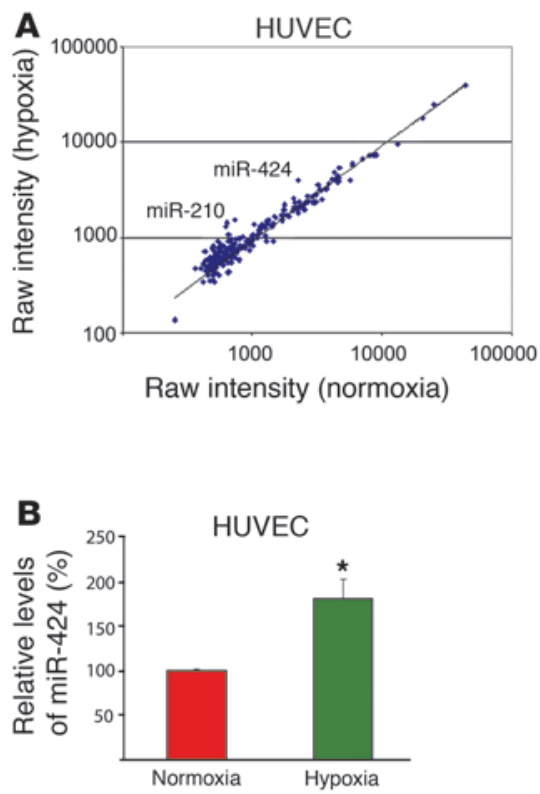
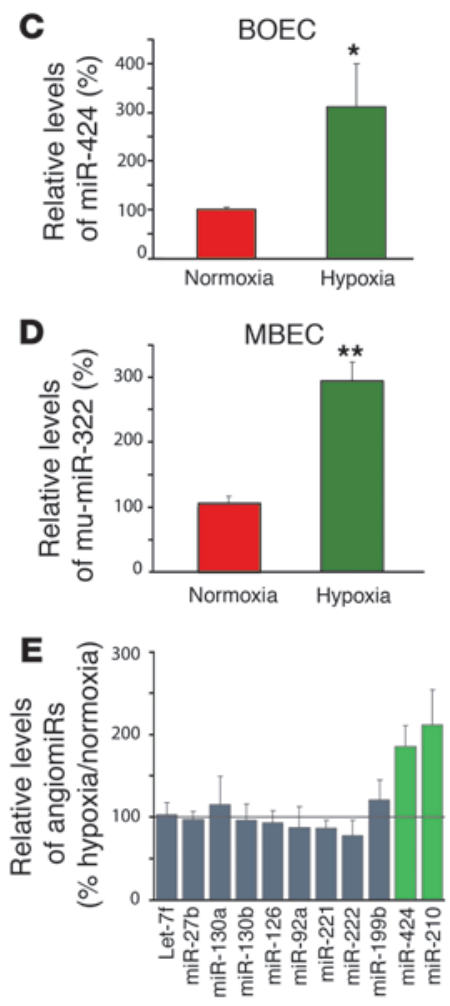

\section{Figure 1}

Hypoxia induces upregulation of miR-424 in ECs. (A) Representative miRNA array data showing relative expression levels of miRNA in HUVECs. Data show raw intensities of individual miRNA under normoxia and hypoxia after 24 hours. (B-D) Hypoxia-induced changes in miR-424/322 were validated by q-PCR. (B) HUVECs, (C) human BOECs, (D) and MBECs. $n=3 .{ }^{*} P<0.05 ;{ }^{* \star} P<0.02$. (E) Relative changes in angiomiRs in HUVECs during hypoxia from miRNA array. $n=4$. Values represent mean \pm SD.

\section{Results}

Vascular ECs are directly in contact with blood, respond immediately to changes in oxygen levels, and depend on VEGF-mediated autocrine stimulation for vascular homeostasis (12). Therefore, we first determined the global changes in miRNA profiles in ECs under normoxia or hypoxia. For these studies, we used primary cultures of HUVECs, microvasculature ECs (MVECs) from foreskin, and circulating blood outgrowth ECs (BOECs).

Hypoxia-induced changes in miRNA expression in ECs. ECs were grown either in normoxia or hypoxia for different periods of time ( 24 or 48 hours) and miRNA levels were determined by custom arrays (13). Details of normalization and statistical methods used to determine changes in expression levels have been previously published (13). For array analyses of the same RNA sample, Pearson correlation coefficients were $0.90-0.99$, demonstrating good reproducibility of our microarray experiments (13). Out of the 720 miRNAs (human, mouse, and rat) analyzed, the majority did not show significant change under hypoxia; however, a small number of miRNAs were either up- or downregulated 2-fold. Four independently derived HUVECs and 3 sets each of BOECs and MVECs were analyzed. These studies showed that miR-210 and miR-424 levels were increased in ECs under hypoxia compared with normoxia after 24 or 48 hours. A representative profile of changes in miRNA levels in HUVECs grown either in normoxia or hypoxia is shown in Figure 1A. Relative expression of miRNAs is also summarized in Supplemental Table 1 (supplemental materials available online with this article; doi:10.1172/JCI42980DS1).

Array data was further confirmed by quantitative real-time RTPCR (q-PCR) to determine changes in miR-424 levels following hypoxia. HUVECs (Figure 1B), BOECs (Figure 1C), and MVECs (Supplemental Figure 1A) showed 2- to 3-fold higher expression of miR-424 under hypoxia compared with normoxia. Similar to human ECs, mouse brain-derived ECs (MBECs) also responded to hypoxia with a 3-fold increase in the mouse homolog of human miR-424, mu-miR-322 (Figure 1D). Next we determined the changes in the expression levels of miRNA that had been previously found to modulate angiogenesis (angiomiRs). Microarray data from 4 independent experiments using HUVECs showed that none of the known angiomiRs changed significantly under hypoxia, except miR-210 and miR-424, which were upregulated (Figure 1E). Previous studies have shown increased miR-210 levels in ECs and tumor cells exposed to hypoxia (14-16). Our studies also confirmed that miR-210 is ubiquitously upregulated in ECs and tumor cell lines, whereas miR-424 is selectively upregulated in ECs and vascular smooth muscle cells (data not shown) under hypoxia. In fact, tumor cells showed either no change or a decrease in miR-424 under hypoxia (data not shown). Therefore, we investigated the physiological role of miR-424 in hypoxic adaptation in vascular ECs and angiogenesis.

miR-424 stabilizes HIF-1 $\alpha$ in ECs. Because HIF-1 $\alpha$ plays a major role in the hypoxia-driven angiogenic response, we further determined the direct role of miR-424 on HIF-1 $\alpha$. For this, a partial human gene containing the mature miR-424 sequence was cloned into a pGSU6 vector. HUVECs were transiently transfected with the miR-424 expressing vector under normoxic conditions. In parallel, cultures were transfected with a control miRNA (miRcontrol). Western blot analyses showed that expression of miR424 increased the levels of HIF-1 $\alpha$ compared with miR-control under normoxic conditions (Figure 2A). ECs are known to express HIF- $2 \alpha$ in addition to HIF- $1 \alpha$. Both are regulated by a similar mechanism that involves proline hydroxylation followed by ubiquitination through the VCBCR complex leading to proteasomal degradation. Therefore, we investigated whether miR-424 expression altered the levels of HIF-2 $\alpha$. Data in Figure 2A show that 


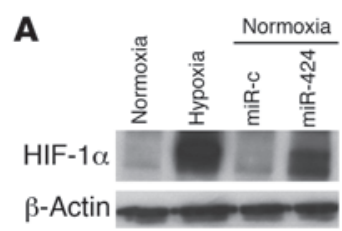

B
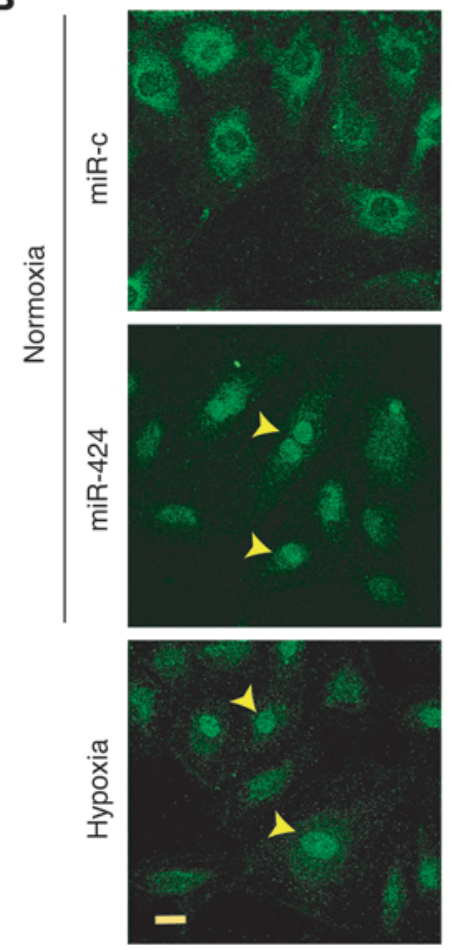

HIF-1 $\alpha$ Actin DAPI

\section{C}

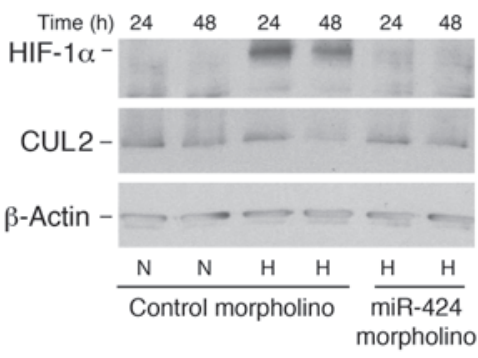

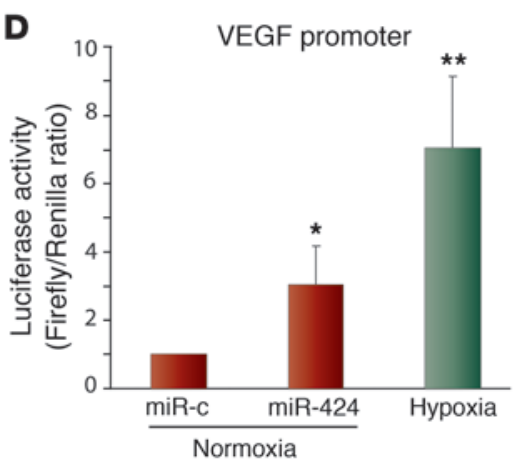

E

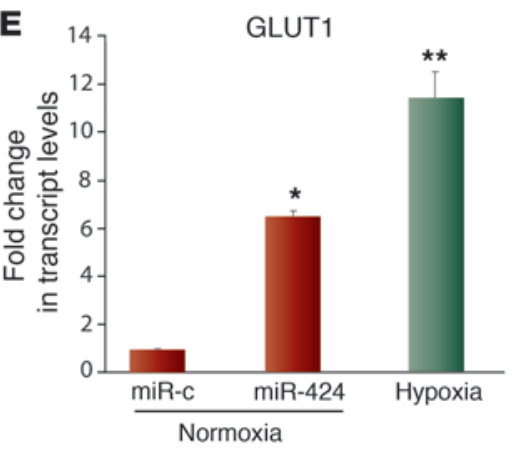

$\mathbf{F}$

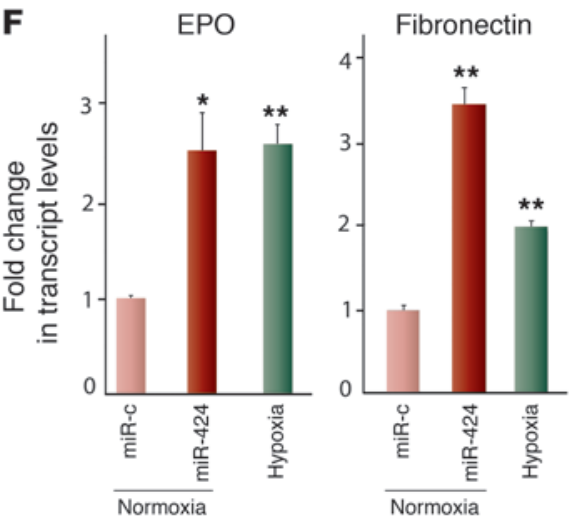

Figure 2

miR-424 stabilizes HIF- $1 \alpha$ and HIF-2 $\alpha$ and increases transcription of HIF- $1 \alpha /$ HIF-2 $\alpha$ target genes. (A) Top: Western blot of HIF-1 $\alpha$ in the wholecell lysates of HUVECs. Normoxia ( $\mathrm{N}$; lane 1 ) and hypoxia $(\mathrm{H}$; lane 2) after 24 hours, and miR-control (lane 3 ) and miR-424 under normoxia (lane 4) after 48 hours. Bottom: Western blot of HIF-2 $\alpha$. miR-c, miR-control. (B) Cellular distribution of HIF-1 $\alpha$ in HUVECs transfected with either miR-control or miR-424 under normoxia. HUVECs exposed to hypoxia were used as a positive control. Arrowheads indicate nuclear accumulation of HIF-1 $\alpha$. Scale bar: $10 \mu \mathrm{m}$. (C) Western blot of HIF-1 $\alpha$ and CUL2 in HUVECs after knockdown of miR-424 under hypoxia. HUVECs treated with control morpholino under normoxia (lanes 1 and 2), hypoxia (lanes 3 and 4), and miR-424-specific morpholino under hypoxia (lanes 5 and 6 ) are shown. (D) HUVECs were cotransfected with either miR-control or miR-424 and a VEGF-luciferase reporter construct under normoxia, or were transfected only with reporter construct and maintained under hypoxia (24 hours). Renilla luciferase levels were used as an internal control. (E and F) HUVECs were transfected with either an miR-control or miR-424 construct and maintained under normoxia. GLUT1, EPO, and fibronectin transcript levels were determined by q-PCR. Values represent mean \pm SD. $n=3 .{ }^{\star} P \leq 0.05 ;{ }^{* \star} P \leq 0.02$.

miR-424 overexpression in HUVECs also increased the levels of HIF-2 $\alpha$. These results indicate that miR-424 expression affects a common pathway by which HIF- $1 \alpha$ and HIF- $2 \alpha$ are stabilized. We next investigated the functional consequence of miR-424-induced increase in HIF-1 $\alpha$. Confocal studies (Figure 2B) showed nuclear translocation of HIF- $1 \alpha$ when miR-424 but not miR-control was expressed in HUVECs under normoxia. ECs exposed to hypoxia were used as a positive control showing nuclear accumulation of 
A

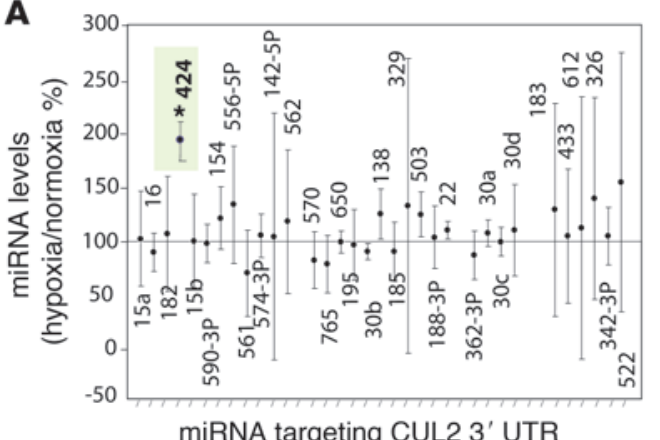

B
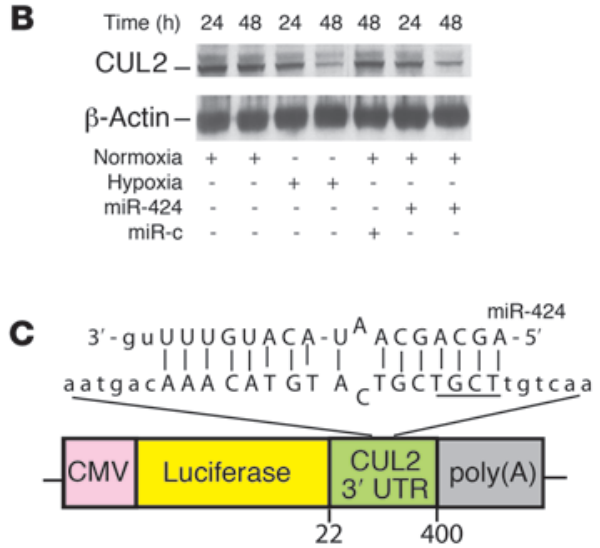
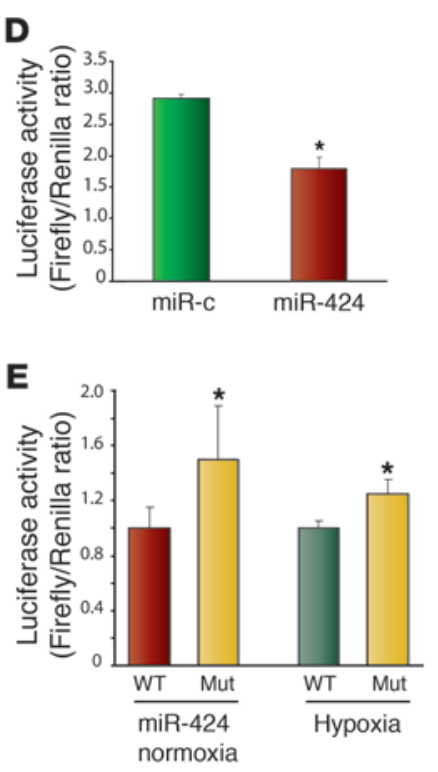

$\mathbf{F}$

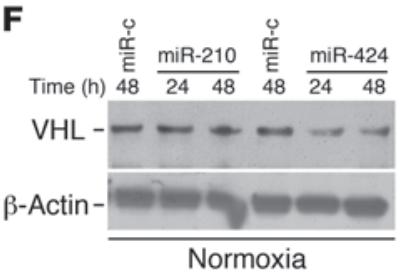

Figure 3

CUL2 is a functional target for miR-424. (A) Microarray data of miRNA expression levels from 4 independent primary cultures of HUVECs were analyzed. Data show relative levels of 37 miRNAs targeting the CUL2 3'UTR under hypoxia. Normoxic levels are considered to be equal to $100 \%$. (B) Western blots showing the expression of CUL2 in the whole-cell lysates from HUVECs under normoxia (lanes 1 and 2) or hypoxia (lanes 3 and 4), miR-control HUVECs under normoxia (lane 5), and miR-424-transfected HUVECs under normoxia (lanes 6 and 7). $\beta$-Actin was used as loading control. (C) Schematic diagram of luciferase reporter construct containing the CUL2 $3^{\prime} U T R$. (D) Luciferase activity (mean \pm SD) in HUVECs cotransfected with the reporter construct of CUL2 3'UTR, miR-424. Renilla luciferase was used as an internal control. (E) Luciferase activity (mean \pm SD) using a reporter construct containing the $3^{\prime} U T R$ of CUL2 (wild-type or mutant lacking the miR-424 target site) under normoxia and hypoxia are shown. (F) Western blot showing the changes in VHL in the whole-cell lysates of HUVECs expressing miR-control (lanes 1 and 4), miR-210 (lanes 2 and 3), and miR-424 (lanes 5 and 6). $\beta$-Actin was used as loading control. Values represent mean \pm SD. ${ }^{*} P<0.05$.

HIF-1 $\alpha$ (Figure 2B). These studies suggest that miR-424 stabilizes HIF- $1 \alpha$ leading to its accumulation in the nucleus. To further confirm above results that miR-424 is involved in the stabilization HIF-1 $\alpha$, we silenced the endogenous miR-424 using specific morpholino. Knocking down miR-424 affected the HIF-1 $\alpha$ stabilization under hypoxia (Figure 2C). These results confirm the that miR-424 is involved in HIF-1 $\alpha$ stabilization in ECs. To determine whether the miR-424-mediated increase in HIF-1 $\alpha$ levels could transactivate target genes under normoxia, we used VEGF promoter-driven expression of luciferase. HUVECs were cotransfected with either miR-424 or miR-control and a VEGF-luciferase reporter construct and were kept under normoxia. miR-424 transfected cells showed 3-fold increase in luciferase activity compared with miR-control-transfected cells (Figure 2D), whereas cells transfected with VEGF-luciferase reporter construct and cultured under hypoxia showed a 7-fold increase in luciferase activity. To further validate miR-424 influence on HIF- $1 \alpha$ transactivation, transcript levels of additional target genes were also determined by q-PCR. miR-424 increased 6 -fold the levels HIF-1 $\alpha$ target genes such as glucose transporter 1 (GLUT1) (Figure 2E). In addition, we determined changes in HIF- $2 \alpha$ target genes as well. HIF- $2 \alpha$ is known to regulate the expression of both erythropoietin (EPO) and fibronectin. miR-424 increased the levels of EPO and fibronectin transcripts by 2.5-fold and 3.7-fold, respectively (Figure 2F). These data suggest that miR-424 regulates the stability of HIF- $1 \alpha / \mathrm{HIF}-2 \alpha$ isoforms and as a consequence increases the expression of their target genes.

CUL2 is a direct and functional target of miR424. To elucidate the mechanism by which miR-424 stabilizes HIF- $1 \alpha$ and HIF- $2 \alpha$, we evaluated potential targets of miR-424 using publicly available algorithms (17-19). These analyses ruled out PHD1-3, FIH, VHL, and elongin $\mathrm{B} / \mathrm{C}$ of the $\mathrm{U} 3$ ligase system involved in the degradation of HIF- $\alpha$. However, CUL2 showed a single complete match for a miR424 target site in its $3^{\prime}$ untranslated region ( $3^{\prime} \mathrm{UTR}$ ). CUL2 is homologous to yeast Cdc53 and is an integral part of VCBCR complex that mediates ubiquitination of HIF- $1 \alpha$ and HIF- $2 \alpha$. Bioinformatics analysis predicted 37 potential miRNA targets in the $3^{\prime} \mathrm{UTR}$ of CUL2 (Figure 3A). miRNA array analyses showed that, of the 37 miRNAs targeting CUL2, only miR-424 levels increased significantly following hypoxia. We therefore hypothesized that miR-424 targets and downregulates CUL2 levels, thereby inhibiting formation of the VCBCR complex. To test this hypothesis, we first checked the protein levels of CUL2 in normoxia and hypoxia and of miR-424-transfected cells under normoxia. These studies showed a substantial reduction in CUL2 protein levels in miR-424-transfected HUVECs maintained under normoxia (Figure 3B). To confirm whether miR-424 can inhibit the synthesis of CUL2, we performed a luciferase reporter assay in which the luciferase reporter gene was fused to the wild-type CUL2 3'UTR (Figure 3C). This assay resulted in $45 \%$ less expression of luciferase activity compared with control when cells were transfected with miR-424-expressing vector (Figure 3D). The binding of miR-424 to the CUL2 3'UTR was specific, as deletion of the seeding region (underlined in Figure 3C) of the miR-424 binding site prevented the reduction in luciferase activity (Figure 3E). Apart from inhibiting translation initiation, miRNAs are known to enhance degradation of target transcripts. Therefore, we investigated whether miR-424 could decrease the levels of CUL2 transcripts. Real-time q-PCR analysis showed a progressive decrease in CUL2 mRNA with time, but the decrease was not statistically significant (Supplemental Figure 2). These results suggest that miR-424 reduces CUL2 
A
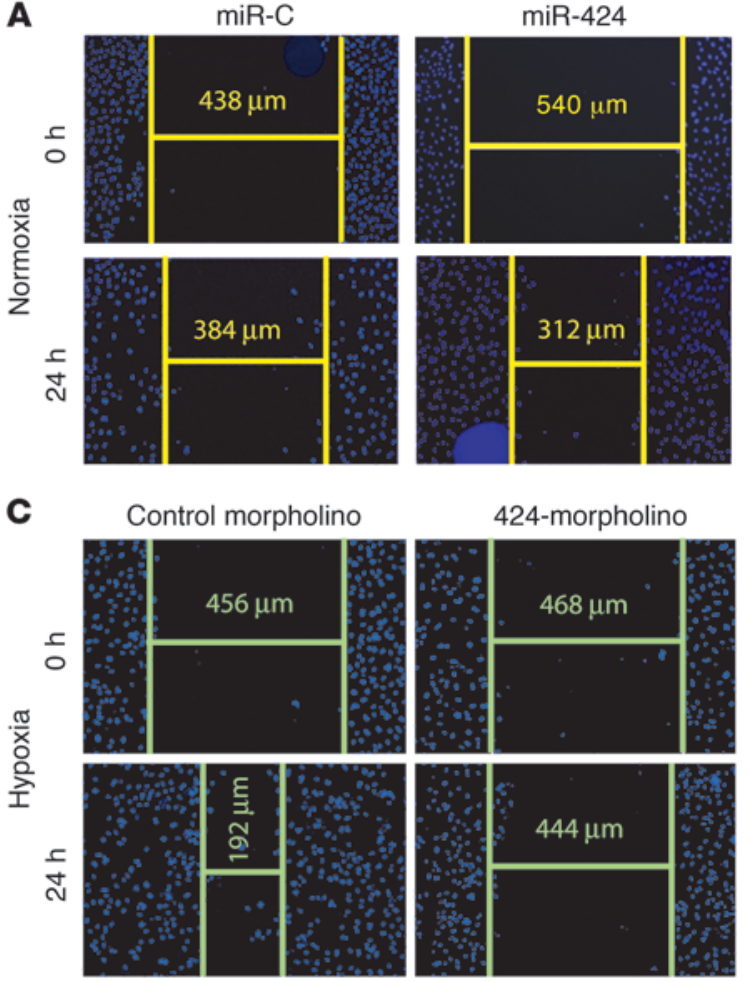

E

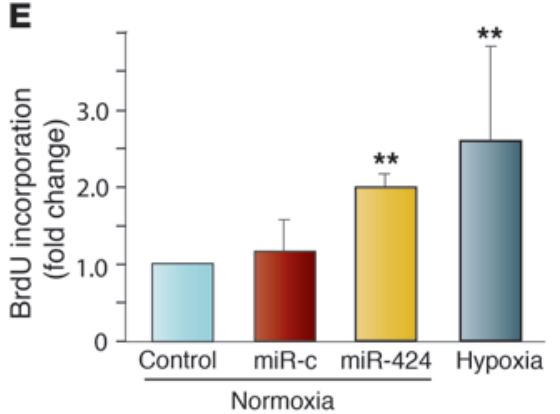

G
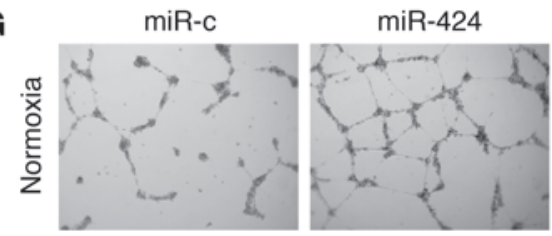

Control morpholino

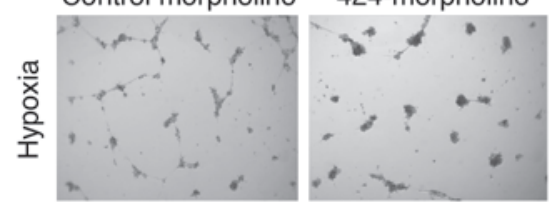

B

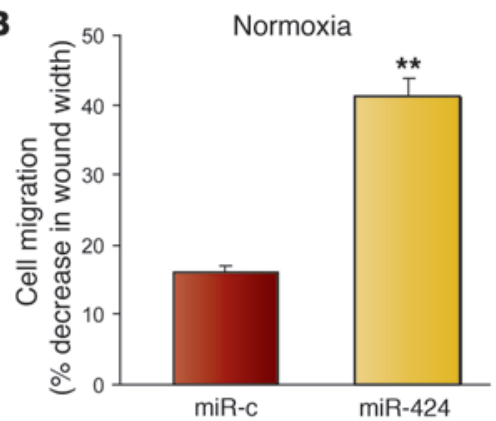

D

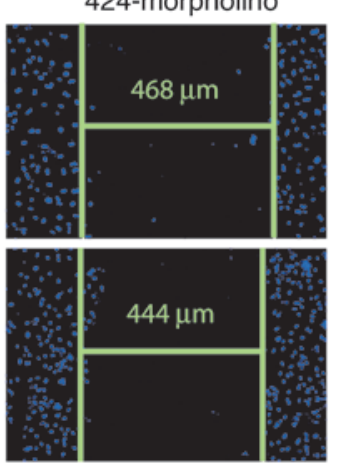

$\mathbf{F}$

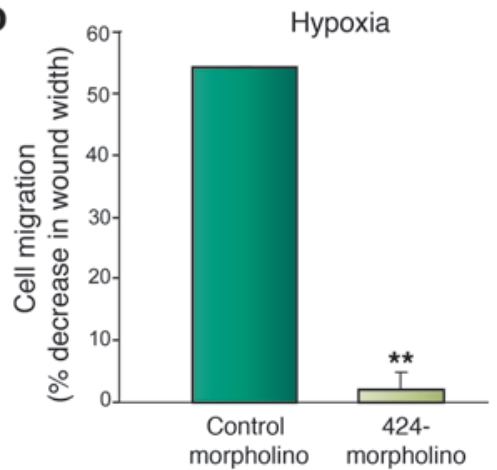

Control

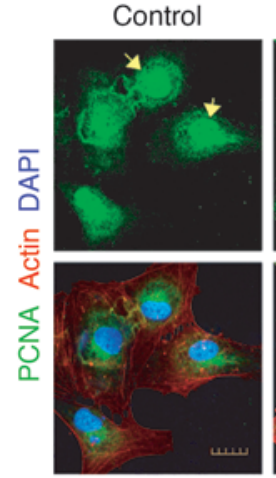
morpholino
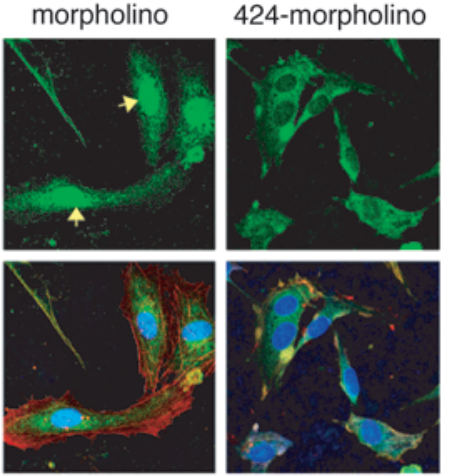

H

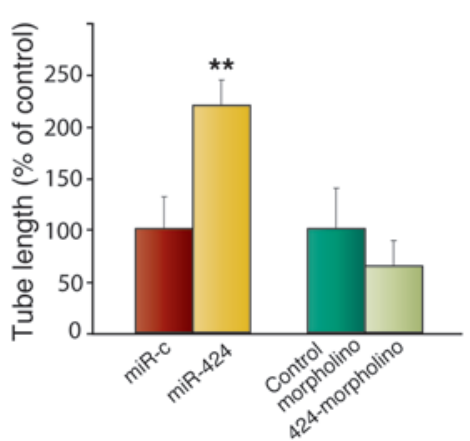

Figure 4

Effect of miR-424 on in vitro angiogenesis. (A and $\mathbf{C}$ ) The effects of miR-424 overexpression under normoxia and silencing by miR424-morpholino under hypoxia on EC migration were determined by scratch wound assay. Wound closure was determined after 24 hours. Yellow and green lines indicate edges of scratch wounds. Representative photomicrographs are shown. Migration of miR-424-overexpressing cells under normoxia (A). Migration of miR-424-morpholino-treated cells under hypoxia (C). (B and D) Quantification of cell migration. (B) Normoxia (miR-control or miR-424 overexpression). (D) Hypoxia (control morpholino or miR-424-morpholino). $n=3$. (E) Effect of miR-424 on cell proliferation. BrdU incorporation was determined by ELISA. Values represent mean \pm SD. $n=3$. (F) Representative confocal images of HUVECs stained for the proliferation marker PCNA. HUVEC cultures were transfected with a control morpholino or miR-424morpholino and grown in media containing $5 \%$ FBS and VEGF (50 $\mathrm{ng} / \mathrm{ml}$ ) under hypoxia. Cells were fixed and stained with anti-PCNA antibody and developed by Alexa Fluor 488-labeled secondary antibody conjugate. Also stained for F-Actin (rhodamine phalloidin: red) and nuclei (DAPI: blue). Scale bar: $20 \mu \mathrm{m}$. (G) Top: Representative photomicrographs of capillary tube formation of HUVECs transfected with miR-control or miR424. Tube formation was scored after 18 hours. Bottom: Tube formation capacity of HUVECs transfected with control morpholino or miR-424-morpholino. The morpholino effect was investigated under hypoxia. Total magnification, $\times 100$. (H) Morphometric analyses of capillary tube length from 3 independent experiments. Values represent mean \pm SD. ${ }^{\star \star} P \leq 0.02$. levels largely by translational inhibition. Furthermore, to validate the role of miR-424 in modulating CUL2 levels, we knocked down the endogenous miR-424 under hypoxia using miR-424-specific morpholino. These studies showed that miR-424-morpholino abrogated the decrease in CUL2 levels under hypoxia, and as a consequence, HIF-1 $\alpha$ stabilization was not observed (Figure 2C).
VHL is the substrate recognition moiety of the multi-protein complex containing CUL2, elongin B/C, and RBX1 (VCBCR), and this complex is responsible for the ubiquitination and degradation of HIF-1 $\alpha$ under normoxic conditions (20). We next determined whether the observed reduction in CUL2 levels by miR-424 could lead to the destabilization of VCBCR components. Western blot 
A

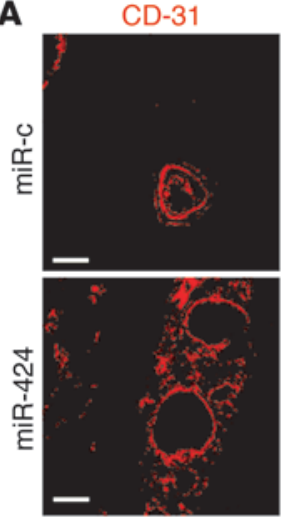

B
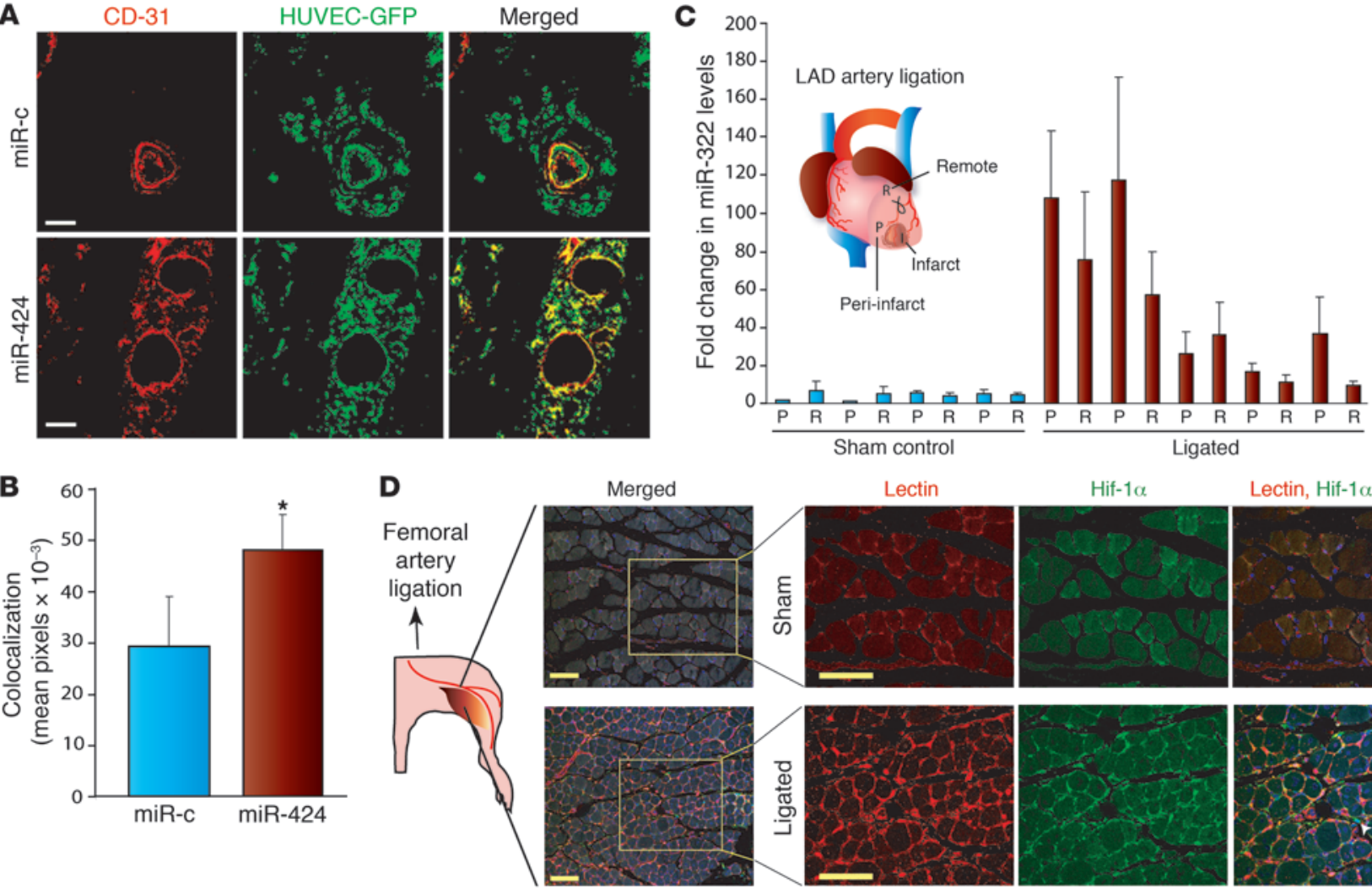

Lectin, Hif-1 $\alpha$, DAPI

$\mathbf{E}$
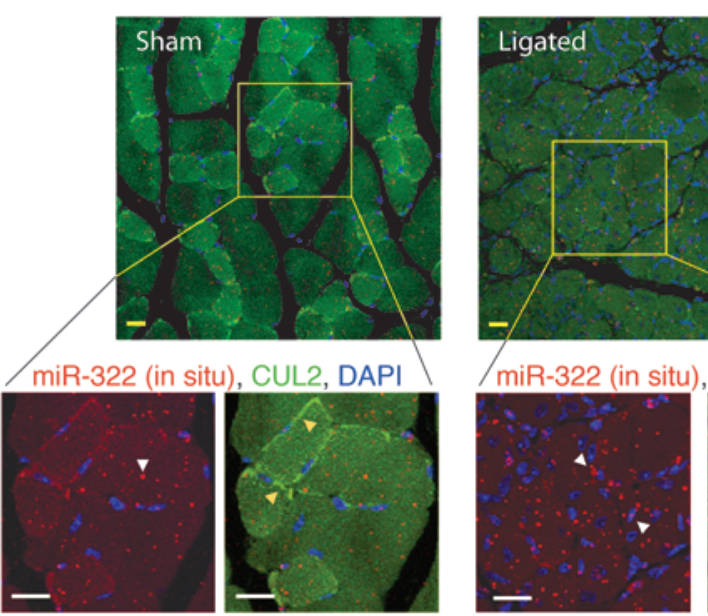

$\mathbf{F}$

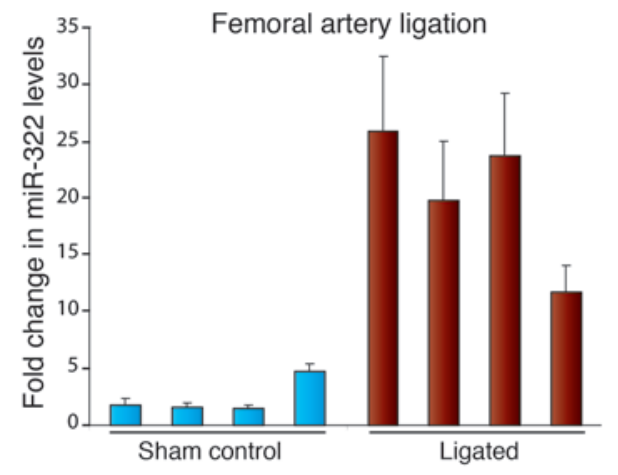

Figure 5

Effect of miR-424 on in vivo angiogenesis. (A) HUVECs were transduced with either GFP-positive miR-control-or GFP-positive miR-424expressing retrovirus. Transduced GFP-positive HUVECs were admixed with ovarian cancer cells in Matrigel and subcutaneously implanted into athymic mice, harvested after 7 days, and processed for vessel staining. Mouse ECs (red), HUVECs (green), and chimeric vessels are shown in merged images. Scale bars: $50 \mu \mathrm{m}$. (B) Metamorph analysis of colocalized pixels of mouse endothelium (red) and HUVECs (green) is shown. Five animals per group and 2 sections of Matrigels (100 $\mu \mathrm{m}$ from the surface) from each sample were analyzed. Ten frames from each section were used to determine the colocalized pixels. (C) Mu-miR-322 levels in rat cardiac tissues from sham-operated and LAD artery-ligated animals. Peri-infarct (P) and remote (R) areas are shown in the schematic diagram. (D) Photomicrographs show representative images of blood vessels and HIF-1 $\alpha$ from the gastrocnemius muscle sections from sham-operated and femoral artery-ligated animals. Vessels were stained with tomato-lectin (red) and HIF-1 $\alpha$ (green). Arrowheads indicate colocalization of HIF-1 $\alpha$ in blood vessels. Scale bars: $40 \mu \mathrm{m}$. (E) Formalinfixed tissue sections from sham-operated and ligated animals were used for in situ hybridization to determine changes in mu-miR-322. Sections were hybridized with miR-322-specific LNA probe (red). The same sections were also stained for CUL2 (green) and nucleus (blue). Scale bars: $10 \mu \mathrm{m}$. (F) q-PCR data of mu-miR-322 levels in the gastrocnemius muscle from sham-operated and ligated animals $(n=3)$. Values represent mean \pm SD. ${ }^{*} P<0.05$ 
A

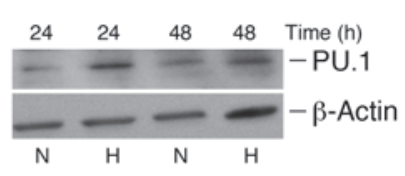

C

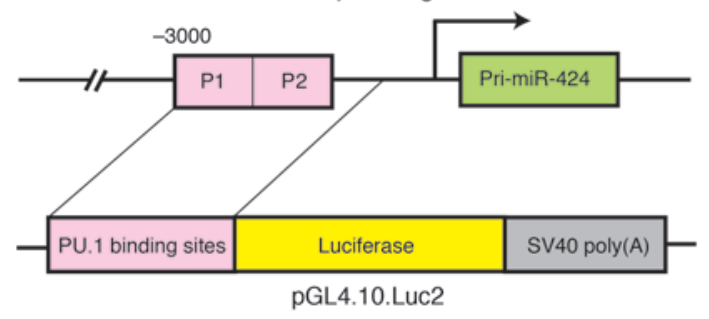

B
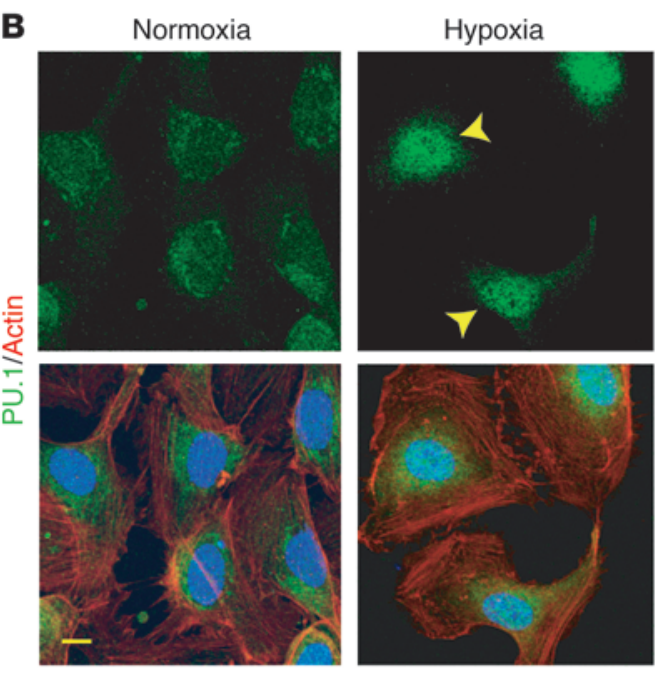
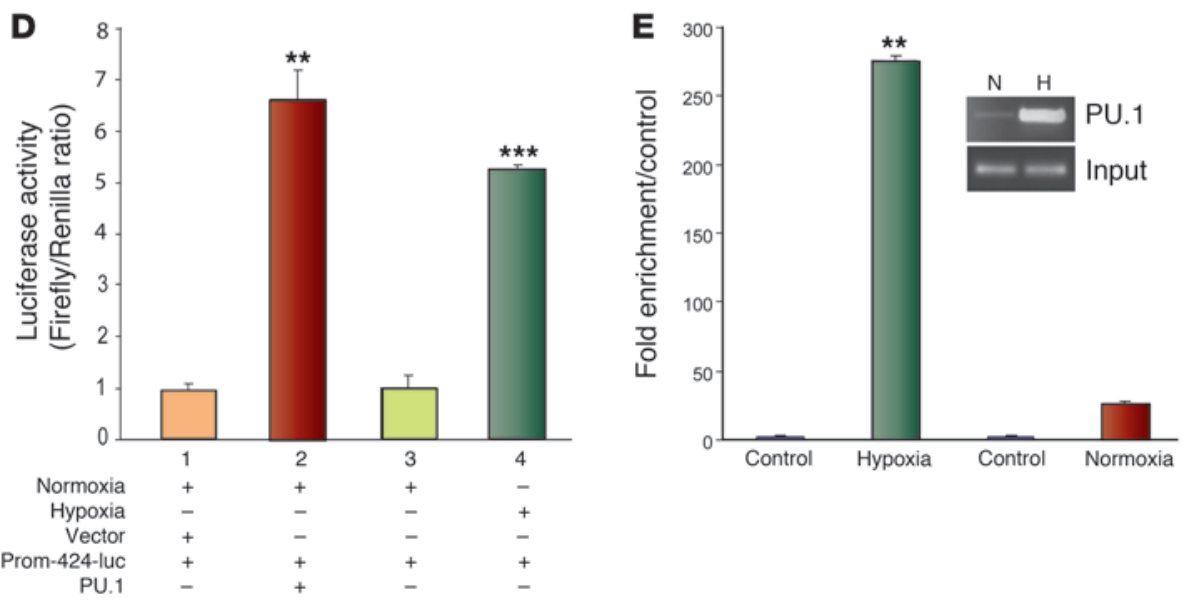

$\mathbf{F}$

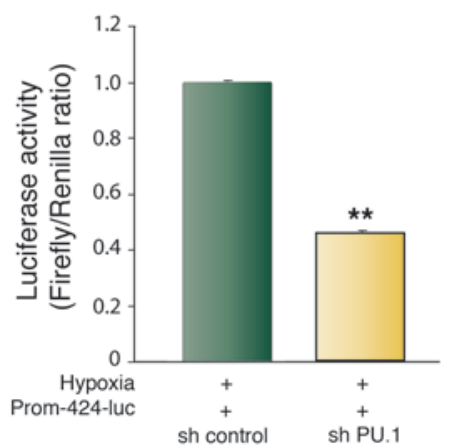

\section{Figure 6}

PU.1 regulates miR-424 expression in ECs. (A) Western blot of PU.1 in the whole-cell lysates from HUVECs under normoxia and hypoxia. $\beta$-Actin was used as loading control. (B) Confocal images of HUVECs stained for PU.1 (green), F-actin (rhodamine phalloidin; red), and nuclei (DAPI; blue). Arrowheads indicate nuclear localization of PU.1. Scale bar: $10 \mu \mathrm{m}$. (C) Schematic diagram of luciferase reporter construct containing the promoter of miR-424, including the putative PU.1 binding sites. (D) Luciferase reporter activity of HUVECs transfected with a construct containing the promoter of miR-424 with putative PU.1 response elements under normoxia (bar 3) or hypoxia (bar 4). HUVECs were cotransfected with either a control vector (bar 1) or with PU.1-expressing vector (bar 2) under normoxia. Cells were also transfected with Renilla luciferase and used as an internal control. (E) ChIP assay. Chromatin preparations were immunoprecipitated with an anti-PU.1 antibody. Recovered DNA was subjected to q-PCR using previously described primers (34). An unrelated genomic region was amplified as a negative control under normoxia or hypoxia. $n=3$. The inset shows representative ChIP blot. $(F)$ Luciferase reporter activity of miR-424 expression after knockdown of PU. 1 under hypoxia. HUVECs were cotransfected with a construct containing the promoter of miR-424 with putative PU.1 response elements under hypoxia with either scrambled control construct (left) or with PU.1-specific shRNA (right). Cells were also transfected with Renilla luciferase, which was used as an internal control. Values represent mean \pm SD. ${ }^{* \star} P<0.02 ;{ }^{* \star *} P<0.001 . n=3$.

analyses showed that miR-424 expression in HUVECs resulted in reduced levels of VHL (Figure 3F) and Rbx1 (Supplemental Figure $3 \mathrm{~A}$ ), whereas neither the miR-control nor miR-210 (which is also upregulated during hypoxia) affected the levels of VHL (Figure 3F) or RBX1 (Supplemental Figure 3A). The role of CUL2 in maintaining the VCBCR complex in normoxia was determined by knocking down endogenous CUL2 by siRNA approach. This experiment showed that reducing the CUL2 levels by using CUL2-specific siRNA under normoxia decreases both RBX1 and VHL1 and thus destabilizes the VCBCR complex (Supplemental Figure 3B). From these data we concluded that miR-424 stabilizes HIF-1 $\alpha$ by targeting CUL2 and thus indirectly destabilizing the components of VCBCR complex.
Effect of miR-424 on in vitro angiogenesis. Vascular sprouting from pre-existing blood vessels requires proliferation of stalk ECs that trail the tip cells that migrate toward a gradient of angiogenic stimulus (21). The primitive plexus thus formed then undergoes tubular remodeling and pruning to allow unidirectional blood flow. Since miR-424 expression increased the levels of HIF-1 $\alpha$ and autocrine stimulation by VEGF can induce ECs, we used 3 in vitro assays to investigate the role of miR-424 in angiogenesis. First, a scratch wound assay was performed to determine the role of miR-424 on cell migration. Results in Figure 4, A and B, show that miR-424 significantly increased cell migration. miR-control-transfected cells narrowed the gap of wounds by $16 \%$, while 

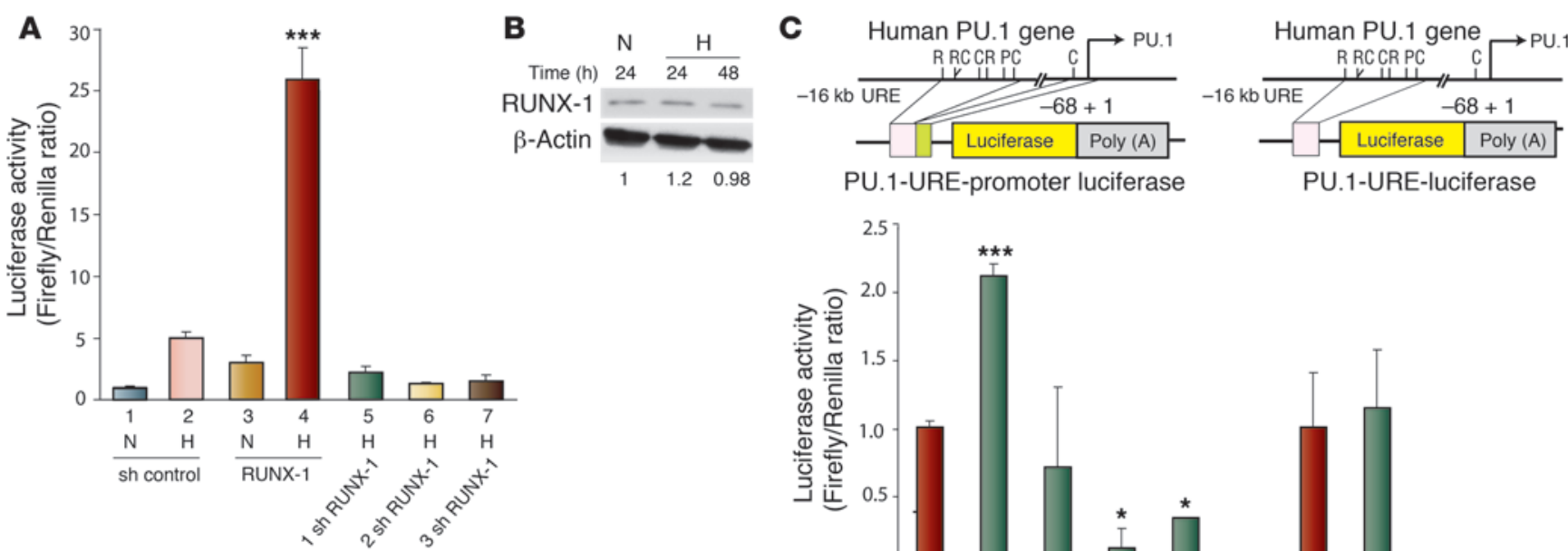

PU.1-URE-promoter luciferase

PU.1-URE-luciferase

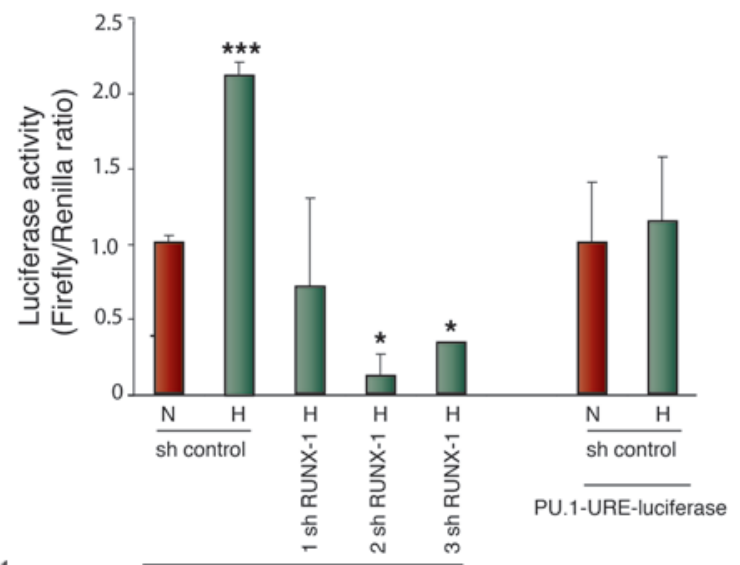

D

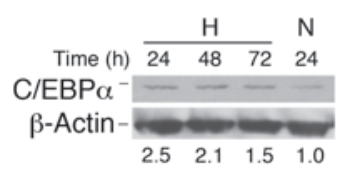

$\mathbf{E}$

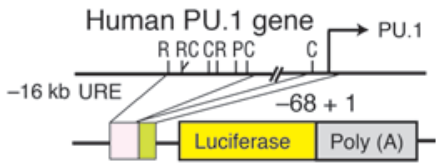

PU.1-URE-promoter luciferase

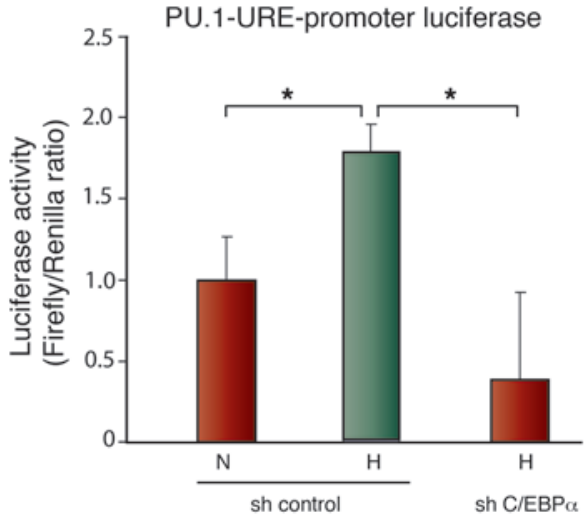

PU.1-URE-promoter luciferase

$\mathbf{F}$

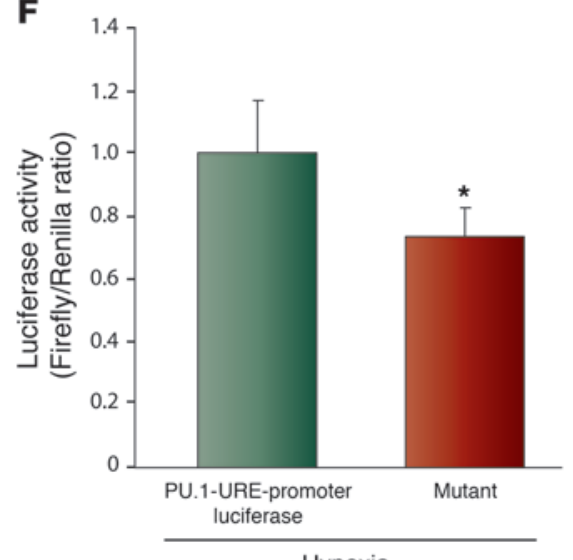

Figure 7

Hypoxia-induced expression of PU.1 is regulated by RUNX-1 (AML1) and C/EBP $\alpha$. (A) HUVECs were transfected with a construct containing the miR-424 promoter, including PU.1 response element and luciferase. Luciferase activity was determined under normoxia (bar 1) or hypoxia (bar 2). Cells were cotransfected with RUNX-1 expression vector, normoxia (bar 3), or hypoxia (bar 4). The effect of RUNX-1-specific shRNAs under hypoxia was determined (bars 5-7). (B) Western blot of RUNX-1 in whole-cell lysates from HUVECs cultured under normoxia or hypoxia. (C) Top: Schematic diagram of luciferase reporter constructs. Bottom: Luciferase reporter activity was measured after cotransfecting HUVECs with the construct containing the PU.1-URE-promoter or PU.1-URE-construct lacking the proximal-68-bp C/EBP $\alpha$ binding site. Cells were cultured under normoxia or hypoxia in the presence and absence of RUNX-1-specific shRNA. Cotransfected Renilla luciferase construct was used as an internal control. Binding sites for RUNX-1, R; C/EBP $\alpha$, C; PU.1, P. (D) Western blot showing increase in C/EBP $\alpha$ levels in HUVECs under hypoxia. (E) Luciferase reporter activity (mean \pm SD) in HUVECs transfected with PU.1-URE-promoter-luciferase (schematic diagram, top) under normoxia or hypoxia in the presence and absence of shRNA to C/EBP $\alpha$. (F) Luciferase reporter activity showing the importance of the C/EBP $\alpha$ binding site at -68 bp of PU.1. HUVECs were transfected with PU.1-URE promoter luciferase construct (left) or a mutant construct lacking the C/EBP $\alpha$ binding site at $-68 \mathrm{bp}$ (right) under hypoxia. Values represent mean $\pm \mathrm{SD}$. ${ }^{*} P<0.05 ;{ }^{* \star *} P \leq 0.001 . n=3$.

miR-424-transfected cells decreased the wound width by $40 \%$. As further proof, we determined the effect of in situ neutralization of miR-424 by specific morpholino under hypoxia. (Figure 4, C and D). Under hypoxia, miR-control cells migrated faster and narrowed the scratch wound with by $38 \%$, whereas in cells transfected with miR-424-morpholino, there was a significant inhibition of migration (2\% narrowing of wound width). Then, we studied the effect of miR-424 on EC proliferation using a BrdU incorporation assay. Data in Figure 4E show an approximately 2 -fold increase in BrdU incorporation in miR-424-transfected HUVECs under normoxia compared with miR-control-transfected HUVECs. Confocal studies showed that proliferating cell nuclear antigen (PCNA), a marker for cell proliferation, was localized in the nucleus of miRcontrol and miR-control-morpholino-treated HUVECs, while 
A

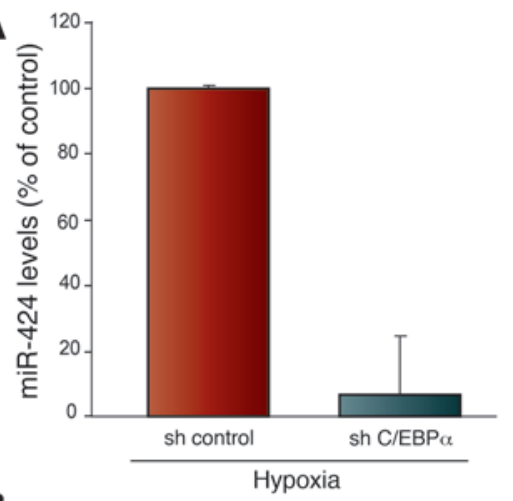

B

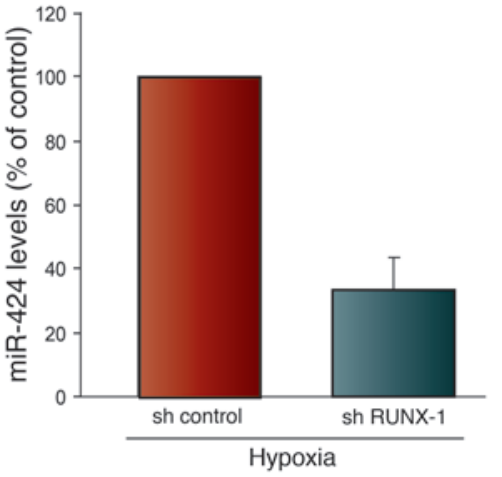

C

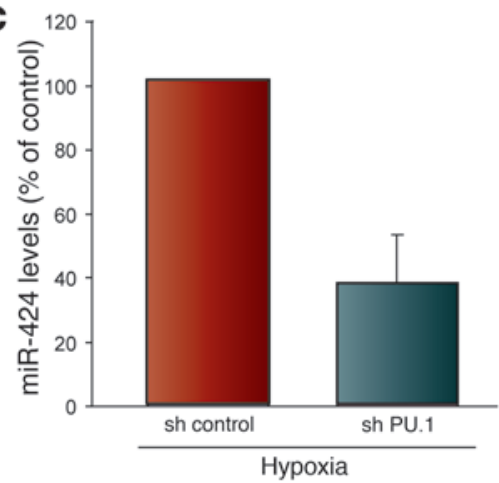

D

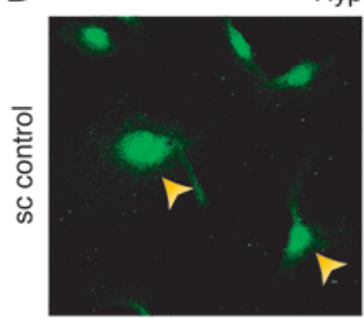

Hypoxia
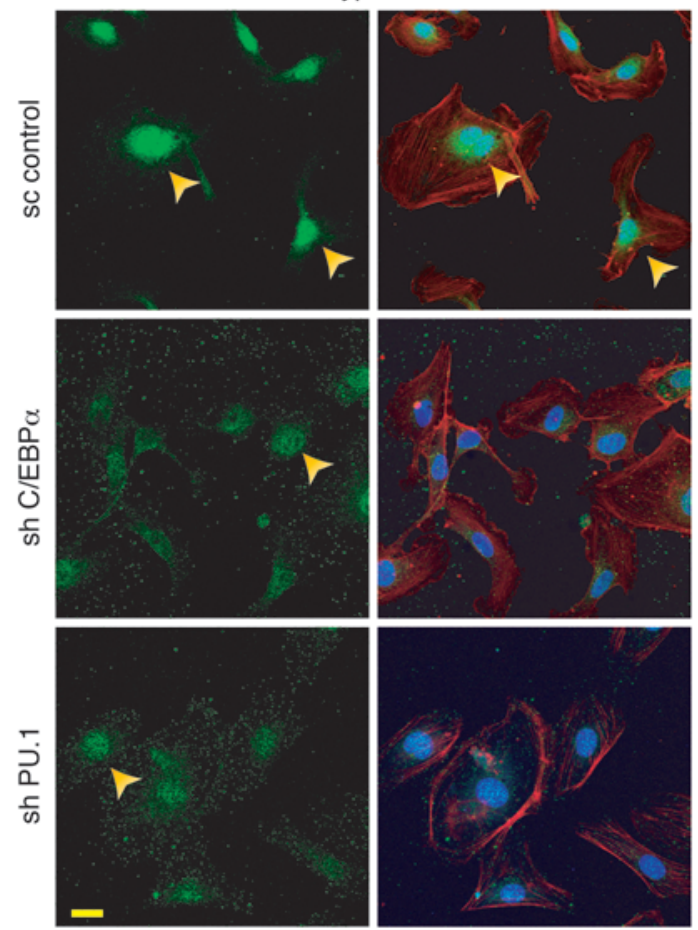

Actin, HIF-1 $\alpha$, DAPI

\section{Figure 8}

Role of PU.1, RUNX-1, and C/EBP $\alpha$ in the regulation of miR-424 expression. (A-C) q-PCR analysis of miR424 expression after knockdown using shRNAs specific to $\mathrm{C} / \mathrm{EBP} \alpha$ (A), RUNX-1 (B), and PU.1 (C) under hypoxia. (D) Representative confocal images showing the effect of shRNAmediated knockdown of C/EBP- $\alpha$ and PU.1 on HIF-1 $\alpha$ nuclear localization. Immunofluorescence studies were carried out by transfecting the cells with shRNA (top), shRNA to C/EBP $\alpha$ (middle), or shRNA to PU.1 (bottom) under hypoxia. Cells were stained for HIF-1 $\alpha$ (green) and nucleus (DAPI; blue). F-actin was stained with rhodamine phalloidin (red). Arrows indicate representative cells showing nuclear localization of HIF-1 $\alpha$. Scale bar: $10 \mu \mathrm{m}$.
miR-424-morpholino treatment inhibited PCNA nuclear localization (Figure 4F and Supplemental Figure 4A) under hypoxia. Finally, we investigated the effect of miR-424 on tube formation. Data in Figure 4, G and H, show that miR-424 increased tube formation in ECs, and that tube formation was inhibited in the presence of miR-424-morpholino. These results suggest that miR-424 can modulate in vitro angiogenesis and that these effects could be negated by miR-424-specific morpholino.

miR-424 and angiogenesis in vivo. We hypothesized that tumor angiogenesis might afford an opportunity to test the role of miR424 in a newly developing microvascular network. We therefore investigated the role of miR-424 in the development of functional vasculature in vivo. To do this, we ectopically expressed miR-424 through retroviral vector MDH1-PGK-GFP-2.0 (22), and HUVECs were transduced with either miR-424- or miR-control-expressing retroviruses. HUVECs expressing miR-424 and GFP (MDH1miR-424-GFP-HUVECs) or miR-control and GFP (MDH1-miRcont-GFP-HUVECs) were admixed with A2780, an ovarian cancer cell line, at a ratio of 1:10 in Matrigel and then subcutaneously implanted into athymic mice. Tumor cells served as an angiogenic stimulus to attract mouse vasculature to sprout and invade the Matrigel containing HUVECs. miR-424-GFP-HUVECs, when implanted in the athymic nude mice, became readily incorporated into the neovessels and colocalized with mouse blood vessels (stained with rat anti-mouse CD31-PE), forming a chimeric vasculature, which indicated that they participated more efficiently in neovascularization than the miR-control-expressing HUVECs (Figure 5A). Metamorph analysis of Matrigel sections showed increased colocalization of miR-424-GFP-HUVECs and anti-mouse CD31-PE signals when compared with miR-controlGFP-HUVECs (Figure 5B). There was no difference in the vascular density of mouse vessels (data not shown), which suggests that the host angiogenic response is not altered between miR-424- and miR-control-expressing HUVEC groups.

Upregulation of rodent miR-322, homolog of human miR-424 in experimental myocardial infarction. Deprivation of cellular oxygen due to impaired perfusion of coronary artery leads to ischemia, infarction, and myocardial remodeling. Ischemia initiates collateral vessel growth, vascular contractility, and erythropoiesis to overcome perfusion deficits. An increase in the steady-state levels of HIF1A 


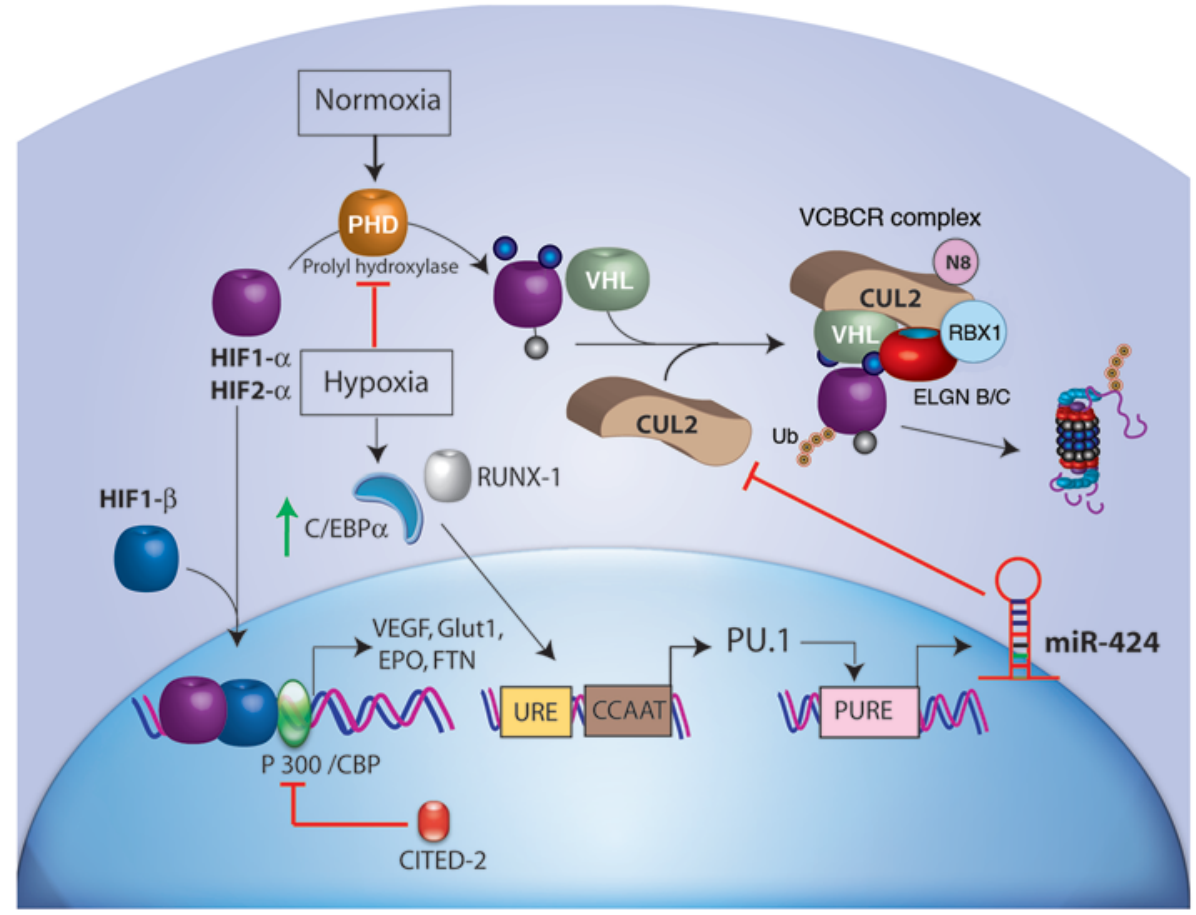

\section{Figure 9}

Schematic diagram showing the regulation of $\mathrm{HIF}-1 \alpha$ by miR-424. C/EBP $\alpha$ levels increase in ECs under hypoxia. C/EBP $\alpha$ in conjunction with RUNX-1 activates the PU.1 promoter. PU. 1 then binds to the miR-424 promoter and induces the expression of miR-424. miR-424 then targets the CUL2 $3^{\prime} U T R$ and inhibits CUL2 expression. A reduction in CUL2 levels leads to the destabilization of VCBCR U3-ligase complex, which leads to the stabilization and nuclear translocation of HIF-1 $\alpha$ in ECs.
mRNA is one of the earliest responses to myocardial ischemia and infarction in humans (23). Rodent models of myocardial infarction are frequently used for experimental studies because the myocardium of the rat, as in humans, has little collateral flow, so occlusion of the left anterior descending (LAD) coronary produces an ischemic zone $(24,25)$. Therefore, we investigated whether miR322 was modulated during experimental myocardial infarction in a rat model. Tissues harvested 1 week following ligation were analyzed for mu-miR-322 levels using q-PCR. Data in Figure 5C show the levels of mu-miR-322 in cardiac tissues. Peri-infarct tissues harvested near the infarct area showed an 18.6-fold increase $(P=0.02)$ in mu-miR-322 levels compared with sham-operated control animals in which the surgical loop was placed around the LAD artery but not tightened, allowing regular blood flow. Interestingly, cardiac tissues obtained from a remote infarct region also showed a significant increase $(7.6$-fold; $P=0.02)$ in mu-miR-322 levels compared with control heart tissues obtained from similar remote sites from sham-operated rats (Figure 5C). These data suggest a physiological association between mu-miR-322/miR-424 and post-ischemic response in myocardial infarction-induced collateral vessel growth and tissue remodeling.

Hindlimb ischemia and mu-miR-322. Peripheral artery disease (PAD) is caused by occlusion or narrowing of large arteries, leading to reduced blood flow to downstream tissues. It is caused by atherosclerosis and inflammatory processes that can cause acute or chronic ischemia in peripheral tissues. Mouse hindlimb ischemia can be experimentally induced by femoral artery ligation, and this model is extensively used to study PADs. The natural response to muscle tissue ischemia includes the mobilization of circulating cellular elements and the upregulation of angiogenic growth factors locally to induce collateral vessel growth (26-28). We used the femoral artery ligation to induce chronic ischemia in gastrocnemius muscle and investigated its effect on HIF- $1 \alpha$, angiogenesis, and mu-miR-322. Control animals were subjected to similar surgical procedures except the ligation step. Figure 5D shows representative images from gastrocnemius muscle sections stained for blood vessels and HIF-1 $\alpha$. Femoral artery ligation resulted in increased capillary sprouting and HIF-1 $\alpha$ expression (Figure 5D). Vessel density increased 3-fold in the ischemic tissue compared with sham control (Supplemental Figure 4B). The higher levels of HIF-1 $\alpha$ in the tissues from ligated animals were confirmed by Western blot, which corroborated the immunohistochemistry results (Supplemental Figure 4, C and D). We further investigated the ischemia-induced changes in mu-miR322 and its effects on CUL2 in vivo via in situ hybridization studies using 3'-DIG-labeled locked nucleic acid (LNA) probes specific to miR-322. Tissues from the ligated experimental group showed higher levels of mu-miR-322 in situ hybridization signal compared with tissues from the sham-operated group (Figure 5E and Supplemental Figure 5). Representative images of mu-miR-322 and CUL2 are shown in Figure 5E. These results clearly demonstrate an inverse correlation between expression of mu-miR-322 and CUL2 levels in ischemic tissues. Furthermore, mu-miR-322 in situ hybridization clearly colocalized with vessels (lectin-FITC), and there was a direct correlation between increased vessel density and increased mu-miR322 expression as seen in the ligated tissues (Supplemental Figure $5 \mathrm{~B})$. Tissues from sham-operated animals showed markedly reduced miR-322 and decreased vessel density (Supplemental Figure 5). q-PCR analysis of the tissues showed an 8.5-fold increase, with a mean of $20.25 \pm 3.12 \mathrm{AU}(P \leq 0.009)$, in mu-miR-322 following limb ischemia compared with sham-operated animals ( $2.4 \pm 0.8 \mathrm{AU}$; Figure $5 \mathrm{~F}$ ). These data again suggest that miR-322/miR-424 is physiologically upregulated in ischemic tissues.

Regulation of miR-424 expression by PU.1 transcription factor in ECs. PU.1 belongs to the family of Ets transcription factors and is expressed predominantly in macrophages, $B$ cells, and neutrophils (29-31). There is growing evidence that hematopoietic stem cells play a critical role in angiogenesis (32). Hemangioblasts are the precursor for both hematopoietic stem cells and ECs, and hypoxia 
is known to regulate hemangioblast differentiation (33). PU.1 was found to regulate miR-424 in human monocyte/macrophage differentiation (34), which prompted us to study whether PU.1 levels are modulated during hypoxia. ECs exposed to hypoxia showed an upregulation and intracellular distribution of PU.1 (Figure 6, A and B). PU.1 was increasingly found in the nucleus under hypoxia with a concomitant decrease in the cytoplasm. These studies suggest PU.1 has a potential role in endothelial response to hypoxia.

To test whether PU. 1 regulates miR-424 in ECs, we constructed a luciferase reporter plasmid containing the promoter of miR-424, including the putative PU.1 response element, PURE, in a promoterless luciferase vector (miR-424-promoter-luciferase) (Figure 6C). Luciferase activity (miR-424 expression) was increased by 6.5 -fold $(P \leq 0.02)$ when HUVECs were co-transfected with a PU.1 expression vector and miR-424-promoter-luciferase construct under normoxia (Figure 6D). HUVECs transfected with the same reporter construct (Figure 6C) showed a 5.25 -fold $(P \leq 0.001)$ increase in luciferase activity when exposed to hypoxia (Figure 6D). Very low luciferase activity was observed when cells were either kept under normoxia or transfected with an empty vector. As additional evidence, ChIP assays were carried out to investigate whether PU.1 binds to the miR-424 promoter under hypoxia. Nucleoprotein complexes prepared from HUVECs grown under either normoxia or hypoxia were immunoprecipitated with an antibody to PU.1. ChIP analysis showed PCR enrichment of a DNA fragment corresponding to the region of PU.1-binding site in the miR-424 promoter. Normoxia showed negligible amplification of the corresponding fragment (Figure 6E). A representative ChIP blot is shown in Figure 6E, indicating the higher levels of PU.1 under hypoxia. PU.1 binding to the miR-424 promoter is specific, since the immunoprecipitates did not amplify control primers corresponding to an unrelated genomic region (Figure 6E). To further validate PU.1 involvement in the regulation of miR-424, shRNAmediated knockdown experiments were carried out. The results showed that knocking down of PU.1 under hypoxia reduced the miR-424 promoter-driven luciferase activity by more than $55 \%$ $(P \leq 0.02)$ when compared with scrambled control (Figure 6F). From these experiments, we concluded that PU.1 interacts with the miR-424 promoter and regulates miR-424 expression in ECs.

Hypoxia-induced expression of PU.1 is regulated by RUNX-1 and $C / E B P \alpha$. The transcription factor RUNX-1 (AML1) is critical for definitive hematopoiesis (35) and is widely expressed in mature hematopoietic cells. Moreover, PU.1 expression is regulated by RUNX-1 in different cell lineages (36). Therefore, RUNX-1 might regulate PU.1 to drive the expression of miR-424 under hypoxia. To this end, we performed a luciferase assay using a miR-424-promoter-luciferase construct (Figure 6C). As demonstrated earlier, we observed a 6 -fold increase in luciferase activity when cells were kept under hypoxic conditions compared with normoxia (Figure 7A). When cells were co-transfected with an expression construct of RUNX-1 under hypoxia, the luciferase activity was further increased (Figure 7A). Thus, RUNX-1 induces the expression of miR-424 by increasing PU.1 expression. We also observed a subtle increase in luciferase activity when the cells were transfected with RUNX-1-expressing vector only. Knockdown of endogenous RUNX-1 by 3 different shRNAs specific to RUNX1 resulted in reduced PU.1 levels in ECs (Supplemental Figure 6) and decreased the activity of the miR-424 promoter (Figure 7A). Next, we evaluated whether RUNX-1 levels change in HUVECs when exposed to hypoxia. Western blot studies showed no change in RUNX-1 levels under hypoxia compared with normoxia (Figure 7B). These results led us to conclude that basal levels of RUNX-1 are important for PU.1-driven expression of miR-424 in ECs.

It is known that RUNX-1 binds to conserved sites in the upstream regulatory elements (UREs) of PU.1 and enhances the expression of PU.1 in hematopoietic cells (36). We then evaluated the role of UREs in PU.1 regulation in ECs as well. For this purpose, we cloned the URE (-16 kb in humans) fragment of the PU.1 gene into a luciferase reporter plasmid (PU.1-URE-luciferase). The URE contained 3 conserved RUNX-1 binding sites, 2 C/EBP $\alpha$ binding sites, and a single PU.1 binding site (Figure 7C). Surprisingly, we did not observe any increase in luciferase activity under hypoxia when this construct was used (Figure 7C). We therefore determined whether $\mathrm{C} / \mathrm{EBP} \alpha$ could transactivate PU.1 expression in ECs. $\mathrm{C} / \mathrm{EBP} \alpha$ is a CCAAT/enhancer-binding transcription factor, which is expressed in the immature cells of granulocytes and monocytes during hematopoiesis $(30,37)$. C/EBP $\alpha$ regulates PU.1 expression by interacting with the PU.1 promoter and -14-kb URE region (mouse) to induce monocyte commitment (38). Moreover, another $\mathrm{C} / \mathrm{EBP} \alpha$ binding site at -68 in the PU.1 promoter is important for PU.1 transcription (39). As we did not detect any increase in PU.1 expression using the PU.1-URE construct, we hypothesized that the PU.1 promoter containing the third C/EBP $\alpha$ binding site at -68 bp is likely responsible for PU.1 expression under hypoxia. To test this, we constructed a luciferase reporter in which the PU.1 promoter, containing the -68-bp region, was ligated to the PU.1-UREluciferase construct (Figure 7C). Using the PU.1-URE-promoter construct, we observed a significant increase in luciferase activity under hypoxia (Figure 7C). The increased luciferase activity was abrogated either by using 3 different shRNAs against RUNX-1 (Figure $7 \mathrm{C}$ ) or by using a shRNA against C/EBPa (Figure 7E). Western blots showed an increase in C/EBP $\alpha$ levels following 24-hour exposure to hypoxia (Figure 7D). In order to confirm that the C/EBP $\alpha$ binding site at $-68 \mathrm{bp}$ in the PU.1 promoter is important for PU.1 expression in ECs, we used a mutant luciferase reporter construct lacking this site. HUVECs transfected with the mutant construct showed reduced PU.1 promoter activity when compared with the cells transfected with the wild-type construct (Figure 7F).

We then examined whether knocking down the levels of C/EBP $\alpha$, RUNX-1, and PU.1 can affect hypoxia-induced expression of miR422 , as direct proof for their regulatory importance. HUVECs were transfected with validated shRNA specific for $\mathrm{C} / \mathrm{EBP} \alpha$ and then exposed to hypoxia. Knockdown of C/EBP $\alpha$ significantly reduced the expression of miR-424 compared to scrambled control transfected cells (Figure 8A). Similarly, knockdown of RUNX-1 and PU.1 with specific shRNA markedly blocked miR-424 expression (Figure 8, B and C). To further evaluate the HIF-1 $\alpha$ stabilization in the C/EBP $\alpha$ - and PU.1-knockdown cells under hypoxia, we used an immunofluorescence assay to determine nuclear localization of HIF-1 $\alpha$. The results from these experiments are summarized in Figure 8D. It is clear from these experiments that HIF- $1 \alpha$ levels were higher in the scrambled RNA control-treated HUVECs under hypoxia and readily localized to the nucleus. In contrast, knockdown of either C/EBP $\alpha$ or PU.1 showed reduced levels of HIF- $1 \alpha$ and its localization to the nucleus. These results suggest that both RUNX-1 and C/EBP $\alpha$ are important for the transcriptional activation of PU.1, even though only C/EBP $\alpha$ is upregulated in a hypoxia-dependent manner. Increased PU.1 then induces the expression of miR-424, leading to stabilization of HIF- $\alpha$ isoforms, and promotes angiogenesis. 


\section{Discussion}

Vascular ECs are in direct contact with blood and respond to lower oxygen levels by increasing HIF- $1 \alpha$, which regulates proangiogenic gene expression and mobilizes $\mathrm{EC}$ progenitors from the bone marrow to the sites of vascular remodeling (40). Major regulation of HIF-1 and HIF-2 $\alpha$ levels in cells under normoxia is controlled by oxygen/VHL-dependent proteasomal degradation $(2,41,42)$ and transcription $(8,41,43)$. Additionally, minor pathways involving RACK1 and histone deacetylase that lead to oxygen/VHL-independent proteasomal degradation of HIF-1 $\alpha$ have been identified (44, $45)$. In the present studies, we provide evidence for a new pathway of HIF- $1 \alpha$ regulation involving miRNA-mediated destabilization of the proteasomal degradation process. However, we determined that miR-424 does not affect the alternate pathways of HIF- $1 \alpha$ stabilization, as we observed further accumulation of HIF-1 $\alpha$ in the presence of the proteasomal inhibitor MG132 (Supplemental Figure 7). ECs from humans and mice showed consistent overexpression of miR-424 mu-miR-322 under hypoxia. Our studies demonstrate that miR-424 targets the $3^{\prime}$ UTR of CUL2 that is conserved in several species including mouse and rat. Among the 37 miRNA that are predicted to target the CUL2 3'UTR, only miR-424 showed a statistically significant increase under hypoxia.

Subsequent studies revealed that overexpression of miR-424 can reduce the levels of CUL2 protein even under normoxia. Luciferase reporter assays confirmed that CUL2 is a valid target for miR-424. As a scaffolding protein, CUL2 plays a crucial role in the assembly of the E3-ligase system (46). miR-424 overexpression reduced the levels of CUL2 transcript and protein (Supplemental Figure 2 and Figure $3 \mathrm{~B})$. These studies suggest that miR-424 can inhibit CUL2 mRNA translation as well as induce its degradation (to a lesser extent). Reducing the CUL2 levels destabilizes the VCBCR complex, resulting in concomitant decrease in VHL and RBX-1. These changes are specific to miR-424 because miR-210, another miRNA upregulated in hypoxia, did not affect CUL2, VHL, or RBX-1 levels. Furthermore, since neither VHL nor RBX-1 has a target site for miR-424, their reduction by miR-424 was likely due to instability imparted by the reduced CUL2 levels. This notion was confirmed by siRNA-mediated silencing of CUL2, which led to decreased levels of RBX-1 and VHL-1 (Supplemental Figure 3B). The VCBCR complex regulates not only the levels of HIF- $1 \alpha$, but also those of HIF- $2 \alpha$. HIF- $2 \alpha$, also known as endothelial PAS domain protein 1 (EPAS), and HIF-1 $\alpha$ are necessary for the optimal functioning of the vascular tissues. HIF- $1 \alpha$ and HIF- $2 \alpha$ govern the expression of distinct and overlapping sets of target genes $(47,48)$. For example, targeted deletion of HIF- $2 \alpha$ in ECs affects Dll4 expression (49), and downregulation of Dll4/Notch signaling is implicated in nonproductive angiogenesis. In this context, it is important to note that miR-424 increases the levels of both HIF- $1 \alpha$ and HIF-2 $\alpha$ by targeting CUL2 (Figure 2A).

ECs transfected with miR-424 construct showed increased transcript levels for the gene targets for HIF- $1 \alpha$ and HIF- $2 \alpha$ such as for VEGF, GLUT-1, and EPO (Figure 2, D-F). These results clearly establish a functional role for miR-424 in HIF- $1 \alpha /$ HIF- $2 \alpha$-mediated gene regulation in energy production, angiogenesis, and erythropoiesis. Moreover, ECs transfected with miR-424 showed a higher rate of proliferation and migration compared with control, possibly a result of autocrine stimulation by HIF- $1 \alpha$-driven VEGF secretion. Morpholino-targeting miR-424 inhibited EC migration and tube formation, confirming miRNA-mediated signaling in the hypoxic adaptation of ECs. During tube formation, a presumptive lumen is formed by vacuolation, a process linked to the CDC42 GTPase signaling pathway involving RAC1 (50). The role of miR-424 in modulating this signaling pathway still needs to be studied.

In addition to in vitro changes in angiogenesis, miR-424 levels were significantly increased during vascular remodeling in vivo. Changes in miR-424 were observed in models of experimental myocardial infarction and PAD. mu-miR-322 was upregulated in peri-infarct cardiac tissues in rats following LAD ligation (Figure 5C). Interestingly, left ventricular tissues harvested away from the infarcted area also showed significantly higher levels of mu-miR-322. These data suggest that ischemia may activate mu-miR-322 expression in the tissues surrounding the site of hypoxic stress as a survival mechanism to initiate collateral vessel growth. It is well established that blood flow changes upstream to a blocked site lead to arteriogenesis due to increased pressure and shear stress affecting the size of the lumen of the blood vessels (51). These changes may have contributed to the observed increase in mu-miR-322 in the remote site as well. Previous studies have shown that following LAD ligation, HIF-1 $\alpha$ levels increase in peri-infarct and remote regions (52). Increased HIF-1 $\alpha$ levels parallel the changes seen in mu-miR-322 in the present study. These results establish a definitive connection between ischemia and mu-miR-322 in the heart during vascular remodeling. In parallel studies using a mouse model of PAD, femoral artery ligation-induced ischemia also led to upregulation of mu-miR-322 in gastrocnemius muscle. HIF-1 $\alpha$ upregulation and collateral vessel growth has been well documented in this model system and has been found to correlate well with clinical settings $(53,54)$. Our studies show that the increase in vessel density following limb ischemia coincided with higher mu-miR-322 levels in muscle. These results further confirm the physiological importance of miR-424/322 upregulation during ischemia.

Several recent studies have shown how miRNAs regulate angiogenesis $(14,55-58)$. miR-126 influences the signaling pathways of VEGFR by targeting PI3K and SPRED1 (57). Knockdown of miR-126, for example, affects vascular integrity and in a subset of surviving $\mathrm{miR} 126^{-/}$, mice higher lethality was found following coronary artery ligation (59). The developmental fate and plasticity of vascular smooth muscle cells are controlled by miR-143 and miR-145 (60). Interestingly, hypoxia did not affect any of these miRNAs, suggesting that miR-126 is likely to be a downstream effector rather than a primary responder to oxygen deprivation. Other studies have shown the involvement of the miR-17-92 cluster in regulating EC functions $(61,62)$. While miR-18a and miR19 a positively regulate tumor angiogenesis, miR-92a was found to inhibit angiogenesis. Antagonizing miR-92a enhanced angiogenic recovery and improved blood flow in mouse models of limb ischemia and myocardial infarction (56). Our studies show that under hypoxia, miR-92a levels decreased by about $10 \%$ in ECs. Two other miRNAs (miR-199a/b and miR-148b) are also consistently downregulated under hypoxia (data not shown). miRNA-199a has been shown to downregulate HIF-1 $\alpha$ in cardiac myocytes (63).

Finally, our studies provide evidence for the mechanism by which miR-424 expression is regulated in ECs during hypoxia. PU.1 has been previously shown to regulate miR-424 expression in myeloid cells (34) and is a major transcription factor involved in myeloid differentiation and B cell development (31). Our studies show for what we believe is the first time that PU.1 levels and nuclear translocation increase in a hypoxia-dependent manner in ECs, and ChIP analysis confirmed PU.1 binding to the promoter of miR-424. PU.1 expression is regulated by the URE located at -14 or $-16 \mathrm{~kb}$ in the mouse or human gene, respectively, and contains 3 RUNX-1 and $1 \mathrm{C} / \mathrm{EBP} \alpha$ binding sites in addition to an autoregulatory PU.1 site. 
RUNX-1 is a weak transcription factor and requires C/EBP $\alpha$ for transcriptional upregulation of PU.1 $(31,36)$. RUNX-1 plays an important function in the ontogeny of hematopoietic progenitor cells during embryonic development (64-66) and controls expression at alternate promoters to determine hematopoietic cell fate (67). Our data show that in addition to hematopoietic tissue, differentiated ECs also constitutively express RUNX-1, and RUNX-1 levels did not change when ECs were exposed to hypoxia. Interestingly, knockdown of RUNX-1 by shRNA affected PU.1 expression under hypoxia and in turn reduced the levels of miR-424. These results imply that RUNX-1 is necessary for miR-424 expression.

$\mathrm{C} / \mathrm{EBP} \alpha$, on the other hand, was upregulated during oxygen deprivation. Promoter analysis has shown that the C/EBP $\alpha$ site proximal to the transcription initiation site $(-68 \mathrm{bp})$ is very important for PU.1 expression (39). The proximal C/EBP $\alpha$ site was found to be critical for PU.1 expression in ECs as well. Reporter expression without the proximal C/EBP $\alpha$ site was not altered under hypoxic conditions, whereas a construct including the proximal site showed increased expression under hypoxia. PU.1 promoter activity was reduced by shRNA directed at either RUNX-1 or $\mathrm{C} / \mathrm{EBP} \alpha$, suggesting that interaction between them is important for hypoxia-induced changes in miR-424 levels. However, we do not yet know how $\mathrm{C} / \mathrm{EBP} \alpha$ levels are increased during hypoxia. Recent studies suggest that RUNX-1 and C/EBP $\alpha$ can physically associate with HIF- $1 \alpha$ to promote HIF- $1 \alpha$-mediated transcriptional activation (68-70). Higher C/EBP $\alpha$ levels combined with enhanced activity could promote PU.1 expression during hypoxia.

Together, our studies show that miR-424 is a critical mediator of oxygen-dependent changes in ECs and is physiologically upregulated in tissues undergoing vascular remodeling and angiogenesis. We propose that ECs respond to hypoxia by upregulating $\mathrm{C} / \mathrm{EBP} \alpha$, which associates with RUNX-1 to activate the PU.1 promoter. Increased PU.1 activates the miR-424 promoter, resulting in increased miR-424. miR-424 targets CUL2, destabilizing the VCBCR U3-ligase complex and increasing levels of HIF-1 $\alpha$ and HIF-2 $\alpha$ (Figure 9). These studies provide evidence for a what we believe is a novel pathway for HIF regulation in ECs during hypoxia/ischemia. As PU.1 is the primary component playing a central role in miR-424 expression, methods to regulate PU.1 and miR-424 expression in ECs may be therapeutically useful.

\section{Methods}

Cells. HUVECs, human MVECs, human BOECs, and MBECs were cultured as previously described (71).

Hypoxia treatment. Cells were maintained under hypoxia by flushing modular incubator chambers (Billups-Rothenberg) for 10 minutes with $95 \% \mathrm{~N}_{2} / 5 \% \mathrm{CO}_{2}$. Under these conditions the $\mathrm{O}_{2}$ levels in the medium were determined to be $3 \%$.

Custom miRNA microarray platform. Custom miRNA microarray experiments and analyses were performed as previously described (72). Microarray data have been deposited to the GEO database (GSE17944).
In vivo angiogenesis. All animal experiment protocols were approved by the IACUCs at the University of Minnesota and VA Medical Center in Minneapolis, Minnesota, USA. HUVECs were transduced with either miR-control- or miR-424-expressing retrovirus. Immunofluorescence analysis showed that more than $70 \%$ cells were GFP positive. q-PCR was carried out to confirm the miR-424 expression in the transduced HUVECs. The HUVEC-expressing miR-control (miR-cont-GFP-HUVEC) or miR-424 (miR-424-GFPHUVEC) were then admixed with an ovarian cancer cell line (A2780) at a ratio of 1:10 in Matrigel. About $400 \mu \mathrm{l}$ of Matrigel containing $4 \times 10^{5}$ cells was then subcutaneously implanted into female, athymic mice. Matrigels were removed after 7 days and snap frozen. Frozen section of the Matrigels were stained with anti-mouse CD31-PE for vessel density measurements (73). The extent to which GFP-positive HUVECs seeded the mouse vasculature was determined by confocal microscopy. About 30 images were taken from each group. Co-localized pixels were calculated by Metamorph software (Molecular Devices, Inc.).

Hindlimb ischemia. The left femoral artery of mice was ligated proximal to the bifurcation into saphenous and popliteal arteries to induce ischemia (74). Sham-operated control animals were subjected to similar surgical protocol, but the femoral artery was not ligated. The mice were euthanized 7 days after ischemia and perfusion-fixed at physiological pressures. The gastrocnemius muscle was surgically removed and paraffin-embedded for immunohistochemistry and miRNA analysis. Vessel length (density) was determined as per previously published methods (73).

Experimental myocardial infarction. The LAD artery of adult, male SpragueDawley rats was ligated to induce myocardial infarction (52). Sham-operated animals were subjected to similar surgical procedures; the ligature was passed around the artery but not tied, thereby allowing normal blood flow. After 1 week, the ischemic heart tissues from the myocardial infarction area, peri-infarct area, and the remote area were snap frozen in liquid nitrogen and used for miRNA expression analysis.

Statistics. Samples for q-PCR were always run in triplicate. All experiments were repeated 3 times. Statistical significance was determined using either 1-way ANOVA or the unpaired Student's $t$ test (1-tailed), depending on the number of experimental groups analyzed.

\section{Acknowledgments}

This work was supported by NIH grants CA114340, DA022935, DA011806, HL081715; a fellowship from NIH training grant T32HL007741 (to G Ghosh); and the Sparboe Endowment for Women's Cancer Research. We thank Jerry Sedgwick and John Oja for assisting in confocal studies, Julia Nguyen for technical support, and Joe DeLarco for his critical comments on the manuscript.

Received for publication March 12, 2010, and accepted in revised form August 25, 2010.

Address correspondence to: Sundaram Ramakrishnan, Department of Pharmacology, University of Minnesota, 6-120 Jackson Hall, 321 Church Street SE, Minneapolis, Minnesota 55455, USA. Phone: 612.626.6461; Fax: 612.625.8408; E-mail: sunda001@umn.edu.

\footnotetext{
1. Fraisl P, Mazzone M, Schmidt T, Carmeliet P. Regulation of angiogenesis by oxygen and metabolism. Dev Cell. 2009;16(2):167-179.

2. Semenza GL. Regulation of cancer cell metabolism by hypoxia-inducible factor 1. Semin Cancer Biol. 2009;19(1):12-16.

3. Wenger RH, Stiehl DP, Camenisch G. Integration of oxygen signaling at the consensus HRE. Sci STKE. 2005;2005(306):re12.

4. Metzen E, Ratcliffe PJ. HIF hydroxylation and cellular oxygen sensing. Biol Chem. 2004;385(3-4):223-230.
}

5. Ohh M, et al. Ubiquitination of hypoxia-inducible factor requires direct binding to the beta-domain of the von Hippel-Lindau protein. Nat Cell Biol. 2000;2(7):423-427.

6. Kaelin WG Jr. The von Hippel-Lindau tumour suppressor protein: $\mathrm{O} 2$ sensing and cancer. Nat Rev Cancer. 2008;8(11):865-873.

7. Clifford SC, Astuti D, Hooper L, Maxwell PH, Ratcliffe PJ, Maher ER. The pVHL-associated SCF ubiquitin ligase complex: molecular genetic analysis of elongin B and C, Rbx1 and HIF-1alpha in renal cell carcinoma. Oncogene. 2001;20(36):5067-5074

8. Kuo HP, Lee DF, Xia W, Wei Y, Hung MC. TNFalpha induces HIF-1alpha expression through activation of IKKbeta. Biochem Biophys Res Commun. 2009; 389(4):640-644.

9. Bartel DP. MicroRNAs: target recognition and regulatory functions. Cell. 2009;136(2):215-233.

10. Dalmay T. MicroRNAs and cancer. J Intern Med. 2008;263(4):366-375.

11. Filipowicz W, Bhattacharyya SN, Sonenberg N. Mechanisms of post-transcriptional regulation by 
microRNAs: are the answers in sight? Nat Rev Genet. 2008;9(2):102-114.

12. Lee $S$, et al. Autocrine VEGF signaling is required for vascular homeostasis. Cell. 2007;130(4):691-703.

13. Zhang X, Xu W, Tan J, Zeng Y. Stripping custom microRNA microarrays and the lessons learned about probe-slide interactions. Anal Biochem. 2009; 386(2):222-227.

14. Fasanaro P, et al. MicroRNA-210 modulates endothelial cell response to hypoxia and inhibits the receptor tyrosine kinase ligand Ephrin-A3. J Biol Chem. 2008;283(23):15878-15883.

15. Chan SY, Loscalzo J. MicroRNA-210: A unique and pleiotropic hypoxamir. Cell Cycle. 2010; 9(6):1072-1083.

16. Chan SY, Zhang YY, Hemann C, Mahoney CE, Zweier JL, Loscalzo J. MicroRNA-210 controls mitochondrial metabolism during hypoxia by repressing the iron-sulfur cluster assembly proteins ISCU $1 / 2$. Cell Metab. 2009;10(4):273-284.

17. Griffiths-Jones S, Grocock RJ, van Dongen S, Bateman A, Enright AJ. miRBase: microRNA sequences, targets and gene nomenclature. Nucleic Acids Res. 2006;34(Database issue):D140-D144.

18. Miranda KC, et al. A pattern-based method for the identification of MicroRNA binding sites and their corresponding heteroduplexes. Cell. 2006; 126(6):1203-1217.

19. Nam S, Kim B, Shin S, Lee S. miRGator: an integrated system for functional annotation of microRNAs. Nucleic Acids Res. 2008;36(Database issue):D159-D164

20. Kamura T, et al. VHL-box and SOCS-box domains determine binding specificity for Cul2-Rbx1 and Cul5-Rbx2 modules of ubiquitin ligases. Genes Dev. 2004;18(24):3055-3065.

21. Carmeliet P, De Smet F, Loges S, Mazzone M. Branching morphogenesis and antiangiogenesis candidates: tip cells lead the way. Nat Rev Clin Oncol. 2009; 6(6):315-326.

22. Chen CZ, Li L, Lodish HF, Bartel DP. MicroRNAs modulate hematopoietic lineage differentiation. Science. 2004;303(5654):83-86.

23. Kim CH, Cho YS, Chun YS, Park JW, Kim MS. Early expression of myocardial HIF-1alpha in response to mechanical stresses: regulation by stretch-activated channels and the phosphatidylinositol 3-kinase signaling pathway. Circ Res. 2002;90(2):E25-33

24. Johns TN, Olson BJ. Experimental myocardial infarction. I. A method of coronary occlusion in small animals. Ann Surg. 1954;140(5):675-682.

25. Selye H, Bajusz E, Grasso S, Mendell P. Simple techniques for the surgical occlusion of coronary vessels in the rat. Angiology. 1960;11:398-407.

26. Folkman J. Angiogenesis in cancer, vascular, rheumatoid and other disease. Nat Med. 1995;1(1):27-31.

27. Losordo DW, Dimmeler S. Therapeutic angiogenesis and vasculogenesis for ischemic disease. Part I: angiogenic cytokines. Circulation. 2004; 109(21):2487-2491.

28. Losordo DW, Dimmeler S. Therapeutic angiogenesis and vasculogenesis for ischemic disease: part II: cell-based therapies. Circulation. 2004; 109(22):2692-2697.

29. Rosenbauer F, Tenen DG. Transcription factors in myeloid development: balancing differentiation with transformation. Nat Rev Immunol. 2007; $7(2): 105-117$.

30. Scott EW, Simon MC, Anastasi J, Singh H. Requirement of transcription factor PU. 1 in the development of multiple hematopoietic lineages. Science. 1994;265(5178):1573-1577.

31. Friedman AD. Transcriptional control of granulocyte and monocyte development. Oncogene. 2007; 26(47):6816-6828

32. Takakura N, et al. A role for hematopoietic stem cells in promoting angiogenesis. Cell. 2000; 102(2):199-209.
33. Ramirez-Bergeron DL, Runge A, Dahl KD, Fehling HJ, Keller G, Simon MC. Hypoxia affects mesoderm and enhances hemangioblast specification during early development. Development. 2004; 131(18):4623-4634.

34. Rosa A, et al. The interplay between the master transcription factor PU.1 and miR-424 regulates human monocyte/macrophage differentiation. Proc Natl Acad Sci U S A. 2007;104(50):19849-19854.

35. Okuda T, Nishimura M, Nakao M, Fujita Y. RUNX1/AML1: a central player in hematopoiesis. Int J Hematol. 2001;74(3):252-257.

36. Huang G, et al. PU.1 is a major downstream target of AML1 (RUNX1) in adult mouse hematopoiesis. Nat Genet. 2008;40(1):51-60.

37. Radomska HS, Huettner CS, Zhang P, Cheng T, Scadden DT, Tenen DG. CCAAT/enhancer binding protein alpha is a regulatory switch sufficient for induction of granulocytic development from bipotential myeloid progenitors. Mol Cell Biol. 1998; 18(7):4301-4314

38. Yeamans C, Wang D, Paz-Priel I, Torbett BE, Tenen DG, Friedman AD. C/EBPalpha binds and activates the PU.1 distal enhancer to induce monocyte lineage commitment. Blood. 2007;110(9):3136-3142.

39. Kummalue T, Friedman AD. Cross-talk between regulators of myeloid development: C/EBPalpha binds and activates the promoter of the PU.1 gene. J Leukoc Biol. 2003;74(3):464-470.

40. Du R, et al. HIF1alpha induces the recruitment of bone marrow-derived vascular modulatory cells to regulate tumor angiogenesis and invasion. Cancer Cell. 2008;13(3):206-220.

41. Maxwell PH, et al. The tumour suppressor protein VHL targets hypoxia-inducible factors for oxygen-dependent proteolysis. Nature. 1999; 399(6733):271-275.

42. Kaelin WG Jr, Ratcliffe PJ. Oxygen sensing by metazoans: the central role of the HIF hydroxylase pathway. Mol Cell. 2008;30(4):393-402.

43. Salceda S, Caro J. Hypoxia-inducible factor 1alpha (HIF-1alpha) protein is rapidly degraded by the ubiquitin-proteasome system under normoxic conditions. Its stabilization by hypoxia depends on redox-induced changes. J Biol Chem. 1997;272(36):22642-22647.

44. Liu YV, Semenza GL. RACK1 vs. HSP90: competition for HIF-1 alpha degradation vs. stabilization. Cell Cycle. 2007;6(6):656-659.

45. Kong X, Lin Z, Liang D, Fath D, Sang N, Caro J. Histone deacetylase inhibitors induce VHL and ubiquitin-independent proteasomal degradation of hypoxia-inducible factor 1alpha. Mol Cell Biol. 2006;26(6):2019-2028

46. Merlet J, Burger J, Gomes JE, Pintard L. Regulation of cullin-RING E3 ubiquitin-ligases by neddylation and dimerization. Cell Mol Life Sci. 2009; 66(11-12):1924-1938.

47. Ratcliffe PJ. HIF-1 and HIF-2: working alone or together in hypoxia? J Clin Invest. 2007;117(4):862-865.

48. Skuli N, Simon MC. HIF-1alpha versus HIF-2alpha in endothelial cells and vascular functions: is there a master in angiogenesis regulation? Cell Cycle. 2009;8(20):3252-3253.

49. Skuli N, et al. Endothelial deletion of hypoxiainducible factor-2alpha (HIF-2alpha) alters vascular function and tumor angiogenesis. Blood. 2009; 114(2):469-477.

50. Iruela-Arispe ML, Davis GE. Cellular and molecular mechanisms of vascular lumen formation. Dev Cell. 2009;16(2):222-231.

51. Lloyd PG, Yang HT, Terjung RL. Arteriogenesis and angiogenesis in rat ischemic hindlimb: role of nitric oxide. Am J Physiol Heart Circ Physiol. 2001 281(6):H2528-H2538.

52. Roy S, Balasubramanian S, Wang J, Chandrashekhar Y, Charboneau R, Barke R. Morphine inhibits VEGF expression in myocardial ischemia. Surgery. 2003;134(2):336-344
53. Ho TK, et al. Increased endogenous angiogenic response and hypoxia-inducible factor-1alpha in human critical limb ischemia. J Vasc Surg. 2006; 43(1):125-133.

54. Bosch-Marce M, et al. Effects of aging and hypoxiainducible factor-1 activity on angiogenic cell mobilization and recovery of perfusion after limb ischemia. Circ Res. 2007;101(12):1310-1318.

55. Kulshreshtha R, et al. A microRNA signature of hypoxia. Mol Cell Biol. 2007;27(5):1859-1867.

56. Bonauer A, et al. MicroRNA-92a controls angiogenesis and functional recovery of ischemic tissues in mice. Science. 2009;324(5935):1710-1713.

57. Fish JE, et al. miR-126 regulates angiogenic signaling and vascular integrity. Dev Cell. 2008;15(2):272-284.

58 . Wurdinger T, et al. miR-296 regulates growth factor receptor overexpression in angiogenic endothelial cells. Cancer Cell. 2008;14(5):382-393.

59. van Solingen C, et al. Antagomir-mediated silencing of endothelial cell specific microRNA-126 impairs ischemia-induced angiogenesis. J Cell Mol Med. 2009;13(8A):1577-1585.

60. Cordes KR, et al. miR-145 and miR-143 regulate smooth muscle cell fate and plasticity. Nature. 2009; 460(7256):705-710

61. Dews M, et al. Augmentation of tumor angiogenesis by a Myc-activated microRNA cluster. Nat Genet. 2006;38(9):1060-1065.

62. Ventura A, Jacks T. MicroRNAs and cancer: short RNAs go a long way. Cell. 2009;136(4):586-591.

63. Rane S, et al. Downregulation of miR-199a derepresses hypoxia-inducible factor-1alpha and Sirtuin 1 and recapitulates hypoxia preconditioning in cardiac myocytes. Circ Res. 2009;104(7):879-886.

64. Chen MJ, Yokomizo T, Zeigler BM, Dzierzak E, Speck NA. Runx1 is required for the endothelial to haematopoietic cell transition but not thereafter. Nature. 2009;457(7231):887-891.

65. Choi K, Kennedy M, Kazarov A, Papadimitriou JC, Keller G. A common precursor for hematopoietic and endothelial cells. Development. 1998;125(4):725-732.

66. Lancrin C, Sroczynska P, Stephenson C, Allen T, Kouskoff V, Lacaud G. The haemangioblast generates haematopoietic cells through a haemogenic endothelium stage. Nature. 2009;457(7231):892-895.

67. Sroczynska P, Lancrin C, Kouskoff V, Lacaud G. The differential activities of Runx1 promoters define milestones during embryonic hematopoiesis. Blood. 2009;114(26):5279-5289.

68. Peng ZG, et al. Physical and functional interaction of Runt-related protein 1 with hypoxia-inducible factor-1alpha. Oncogene. 2008;27(6):839-847.

69. Jiang $Y$, et al. Desferrioxamine induces leukemic cell differentiation potentially by hypoxia-inducible factor-1 alpha that augments transcriptional activity of CCAAT/enhancer-binding proteinalpha. Lenkemia. 2005;19(7):1239-1247.

70. Janardhan HP. The HIF-1 alpha-C/EBP alpha axis. Sci Signal. 2008;1(43):jc2.

71. Lin Y, Chang L, Solovey A, Healey JF, Lollar P, Hebbel RP. Use of blood outgrowth endothelial cells for gene therapy for hemophilia A. Blood. 2002; 99(2):457-462.

72. Kalscheuer S, Zhang X, Zeng Y, Upadhyaya P. Differential expression of microRNAs in early-stage neoplastic transformation in the lungs of F344 rats chronically treated with the tobacco carcinogen 4-(methylnitrosamino)-1-(3-pyridyl)-1-butanone. Carcinogenesis. 2008;29(12):2394-2399.

73. Wild R, Ramakrishnan S, Sedgewick J, Griffioen AW. Quantitative assessment of angiogenesis and tumor vessel architecture by computer-assisted digital image analysis: effects of VEGF-toxin conjugate on tumor microvessel density. Microvasc Res. 2000; 59(3):368-376

74. Basi DL, et al. Femoral artery neointimal hyperplasia is reduced after wire injury in Ref-1+/- mice. Am J Physiol Heart Circ Physiol. 2007;292(1):H516-H521. 